\title{
Entwicklungsstörungen des Gehirns und Epilepsie.
}

\section{Zugleich ein Beitrag zur pathologischen Anatomie und Genese der Heterotopien grauer Substanz.}

(Aus dem Pathologischen Institut des Allgemeinen Krankenhauses HamburgEppendorf [Prof. E. Fraenkel] und aus der Kgl. Universitäts-Nervenklinik zu Halle a. S. [Geheimrat Anton].)

Von

Dr. Friedrich Wohlwill.

Mit 3 Textfiguren und 2 Tafeln.

(Eingegangen am 11. März 1916.)

Wenn man mit Recht behaupten kann, daß es heutzutage wohl kaum noch vorkommen wird, daß bei der pathologisch-anatomischen Untersuchung des Gehirns eines epileptischen Individuums Abweichungen von der Norm völlig vermißt werden, so sind doch die hier zu erhebenden Befunde so mannigfaltiger Art und von so verschiedener Dignität, daß es geboten erscheint, eine Sichtung vorzunehmen und sich über die Bedeutung der einzelnen Erscheinungen völlig klar zu werden. In diesem Sinne kann man, wie mir scheint, - wenn wir von ganz zufälligen Nebenbefunden, die mit den epileptischen Anfällen gar nichts zu tun haben, absehen - vier Gruppen pathologischer Gehirnveränderungen unterscheiden.

- Die erste Gruppe würde diejenigen Prozesse betreffen, die wir als direkte Ursache der Anfälle zu betrachten Anlaß haben. Es handelt sich hier meistens um gröbere anatomische Veränderungen (Tumoren, Narben, usw.), die durch einen chronischen Reiz oder indirekt durch ihre Wirkung auf den Hirndruck und dgl. epileptische Anfälle auslösen. Derartige Fälle als solche symptomatischer Epilepsie von denen $\mathrm{zu}$ trennen, die wir als idiopathische, genuine, echte usw. zu bezeichnen pflegen, sind wir meines Erachtens auch heute noch berechtigt, auch dann, wenn die in Betracht kommenden Veränderungen weniger grober Natur sind, z. B. in einer Reichardtschen Hirnschwellung bestehen.

In die zweite Gruppe von Veränderungen würden diejenigen gehören, die wir als anatomisches Korrelat des Anfalls resp. als die Anzeichen des bei jedem Anfall stattfindenden Zerfalls von Nervensubstanz zu betrachten haben. Es sind das die namentlich 
von Alzheimer nachgewiesenen akuteren Veränderungen an den Ganglienzellen und an der Glia - letztere im Sinne des Amöboidismus mit den zugehörigen besonderen Abbauvorgängen -, wie wir sie im Gehirn von Individuen finden, die im Anfall resp. im Status epilepticus zugrunde gegangen sind.

In die dritte Gruppe pathologischer Befunde würden diejenigen zu verweisen sein, die als Folge, und zwar als Dauerfolge zahlreicher Anfälle zu betrachten sind, nach heutiger Anschauung also neben dem Schwund von Ganglienzellen vor allem die Randgliose, vielleicht auch die Ammonshorn-Sklerose (Alzheimer).

An vierter Stelle endlich wären solche Abweichungen von der Norm zu nennen, die weder als Ursache, noch als Folge der Anfälle zu betrachten sind, die vielmehr nur eine Prädisposition des betreffenden Gehirns zur Erkrankung an Epilepsie dokumentieren. Es sind das in erster Linie alle jene mannigfachen Anomalien im Aufbau des Zentralorgans, die auf einer gestörten Entwicklung beruhen, mag diese letztere nun durch Vererbung im Keim oder durch früh - d. h. intrauterin oder in den ersten Lebensjahren - erworbene Krankheiten, Traumen und dgl. bedingt sein.

Gewiß ist es heute noch nicht möglich, die Zugehörigkeit jedes einzelnen pathologischen Symptoms bestimmt festzulegen. Ich erinnere nur an die verschiedene Auffassung von der Bedeutung der Randgliose (Chaslin, Alzheimer, Jakob!), die von anderen vielleicht nicht in die dritte, sondern in die vierte oder auch in die erste Gruppe gerechnet werden würde. Auch von den Residuen kindlicher Encephalitiden, die doch eine so wichtige Rolle in der Vorgeschichte der Epilepsie spielen, dürfte es zweifelhaft sein, ob sie der ersten oder der vierten Gruppe zugehören. Ich bin aber überzeugt, daß diese Schwierigkeiten mehr in unserer Unkenntnis ihren Grund finden, als etwa darin, daß hiér wirklich Utbergänge bestünden; und jedenfalls scheint mir das Bestreben nach reinlicher Scheidung berechtigt.

Hier nun soll von den zur vierten Gruppe gehörigen pathologischen Erscheinungen die Rede sein, und zwar von solchen, die einer bereits intrauterin entstandenen Entwicklungsstörung ihre Entstehung verdanken. Daß solche Entwicklungsanomalien in Gehirnen von Epileptikern durchaus nichts Seltenes sind, unterliegt wohl keinem Zweifel. So weiß man schon seit langer Zeit, daß Heterotopien grauer Substanz bei Epileptikern häufig zu finden sind, und schon einer der ersten der hierher gehörigen Fälle - der von Meschede betrifft einen Epileptiker. Auf feinere Abweichungen von der Norm, die dafür aber um so häufiger sind, ist man erst in neuerer Zeit aufmerksam geworden. Ich erinnere an die Befunde von Ranke, Alzheimer, Turner, Roncoroni, Bevan-Levis, Jakob. Ich brauche 
auf dieselben hier nicht noch einmal zurückzukommen, weil Jakob sie am Schluß seiner Arbeit kurz zusammengestellt hat. Ich beabsichtige vielmehr, zwei eigene, untereinander recht verschiedenartige Fälle etwas ausführlicher mitzuteilen und zum Schluß an ihrer Hand die Bedeutung solcher Befunde für die Klassifikation der Epilepsiefälle zu erörtern.

In dem ersten von mir beobachteten Fall handelt es sich um die bei der Kran. kenhausaufnahme $1^{3} / 4$ Jahre alte Tochter eines gesunden Arbeiters, Carla M., deren Mutter als Kind Krämpfe gehabt hatte und späterhin ein sehr dürftiges, schwächliches Geschöpf geworden war. Von den 7 Geschwistern der Pat. sind 3 an Lebensschwäche resp. an Keuchhusten gestorben, die übrigen 4 sollen sämtlich sehr schwächlich - angeblich lungenkrank - sein. Keine Aborte der Mutter. Kein Anhalt für Syphilis oder Alkoholismus bei den Eltern. Die Geburt unserer Pat., der dritten in der Reihenfolge der Kinder, erfolgte nach normal verlaufener Schwangerschaft $1 \frac{1}{2}$ Monate zu früh. Es soll sehr viel Fruchtwasser vorhanden gewesen sein. Sonst verlief der Partus ohne Zwischenfälle. Das Kind wog bei der Geburt $2 \mathrm{~kg}$ und blieb auch weiterhin sehr schwächlich. Immerhin bot es bis zum Alter von $3 / 4$ Jahren keine besonderen Krankheitszeichen. Dann erkrankte es an schwerem Brechdurchfall und lag 1/4 Jahr im Krankenhaus. Als es zurückkam, war es "gesund" bis auf starke Zeichen ,englischer Krankheit". Im Alter von $5 / 4$ Jahren traten zum ersten Male Kram pfanfälle von der alsbald zu schil. dernden Art auf. Dieselben nahmen an Intensität und Häufigkeit allmählich zu, weswegen am 7. August 1912 die Aufnahme in das Eppendorfer Krankenhaus stattfand.

Was die körperliche und psychische Entwicklung des Kindes bis zum Ein. setzen der Krämpfe betrifft - daß dieselbe von da an keine weiteren Fortschritte machte, entspricht sonstigen Erfahrungen -, so konnte es nach den Angaben der Mutter zu jenem Zeitpunkt, also mit $5 / 4$ Jahren, weder gehen noch stehen, noch auch nur sitzen; dagegen konnte es den Kopf halten. Es war noch nicht reinlich und hatte erst 4 Zähne. Bei der Erklärung dieser somatischen Symptome ist natürlich die schwere Rachitis sehr zu berücksichtigen. Anfänge zum Sprechen hatte das Kind noch nicht gemacht; ob Sprachverständnis vorhanden war, konnte ich von der Mutter nicht mit Sicherheit erfahren. Das Kind griff nach Gegenständen, horchte bei Geräuschen auf, hatte also sicher Gesichts- und Gehörs e mpfind ungen. Im übrigen war der Mutter nur noch aufgefallen, daß es nicht nur, wie andere Kinder, alle Gegenstände wahllos in den Mund steckte, sondern andererseits auch EBbares anscheinend nicht als solches erkannte und z. B. mit Schokolade und Keks wischende Bewegungen machte, ohne sie zu sich zu nehmen. Wir werden aber hierauf keinen zu großen Wert legen dürfen, zumal wenn wir die weitere Angabe der Mutter für richtig halten wollen, daß das Kind seine Eltern erkannt, d. h. von Fremden unterschieden habe, was ja eine viel höhere psychische Leistung vorstellen würde.

Bei der Aufnahme ins Eppendorfer Krankenhaus ${ }^{1}$ ) (7. VIII. 1912) befand sich das Mädchen in leidlich gutem Ernährungszustand. Haut und Schleimhäute waren ziemlich blaß. Es fanden sich die Zeichen schwerer Rachitis: stark aufgetriebene Epiphysen, ausgesprochener „Rosenkranz", weit offene Fontanellen. Im übrigen ergab die körperliche Untersuchung weder an den inneren

1) Für die gütige Überlassung der Krankengeschichte sage ich den Herren Prof. Brauer, Oberarzt Dr. Kissling und Prof. Schottmüller meinen besten Dank. 
Organen noch am Nervensystem irgendeinen pathologischen Befund, insbesondere fanden sich keinerlei Anzeichen für eine Tetanie.

Die Krämpfe, die das Kind anfangs nur selten, späterhin täglich hatte, bestanden in Kontraktionen in allen vier Extremitäten, teils tonischer, teils klonischer Natur. Bisweilen schlossen sich daran Zuckungen im linken Facialis an. Vor und bei den Anfällen schrie das Kind laut. Völlige Bewußtlosigkeit schien nicht zu bestehen. Die Pupillen reagierten. Keine Enuresis. Nach dem Anfall Schaum vor dem Munde. Dauer des Anfalls einige Sekunden bis mehrere Minuten. Die Anfälle traten vorzugsweise nachts, aber auch am Tage auf.

Im Laufe der Beobachtung häuften sich die Anfälle immer mehr, sie wurden durch Phosphorlebertran und Seesalzbäder gar nicht, durch Brom in geringem Maße beeinflußt. In der anfallsfreien Zeit war Pat. anfangs ganz munter, späterhin aber ziemlich schläfrig. Facialisphänomen und Troussea usches Symptom wurden bei häufiger Prüfung stets negativ befunden. Am 19. X. 1912 erkrankte Pat. an einem schweren Scharlach, dem es schon nach 3 Tagen im Alter von nicht ganz 2 Jahren erlag.

Die Sektion wurde am folgenden Tage vorgenommen. Die anatomische Diagnose lautete: Rachitis, teilweise in Heilung; Bronchopn. lob. sup. dextr. Intumescentia follicul. pharyngis. Hyperaemia pharyngis. Intusmesc. adiposa renum. Hyperaemia cerebri.

Uns interessiert hier nur der Befund am Gehirn, das mir von Herrn Prof. Fraenkel gütigst zur Untersuchung überlassen wurde. Das Organ zeigt äußerlich kein auffallende Abweichung von der Norm; nur waren die Windungen zum Teil etwas plump, nicht so fein gegliedert wie normal. Im übrigen aber entsprach die Konfiguration der Oberfläche vollkommen dem gewohnten Bild. Nachdem das Gehirn nach der Virchowschen Technik seziert worden war, zeigte sich, daß die Rindensubstanz - wie wir das bei Scharlachgehirnen fast ausnahmslos finden - hochgradig hyperämisch war. Durch diese Eigentümlichkeit fiel ein Befund ganz besonders stark in die Augen, nämlich die ungewöhnliche Breite der Hirnrinde. Dieselbe war viel zu allgemein, als daß man sie durch Schrägschnitte hätte erklären können. Bei genauerer Untersuchung konnte man dann an vielen Windungen innerhalb der grauen Substanz einen feinen parallel zur Oberfläche ziehenden weißen Streifen erkennen, der diese verbreiterte Rinde in zwei Schichten teilte.

Es wurde nun, da der Befund in beiden Hemisphären identisch erschien, die eine Hemisphäre in toto eingelegt und so gut es an dem bereits sezierten Gehirn ging, zu Serien von Frontalschnitten verarbeitet, die nach Weigert$P a l$ und nach van Gieson gefärbt wurden. Von der anderen Hemisphäre wurden kleinere Blöcke in 96 proz. Alkohol, in Formol und in Gliabeize fixiert und die Schnitte nach allen üblichen Färbemethoden (insbesondere: mit Toluidinblau, Mannscher Methode, Spielmeyers Markscheidenfärbung, Bielschowskys Fibrillenimprägnation, Weigerts Gliafaserfärbung usw.) behandelt ${ }^{1}$ ).

Die Frontalserien lassen erkennen, daß die hier in Betracht kommende Störung fast über das ganze Großhirn verbreitet ist. Nur die Ammons-

1) Leider sind Hirnstamm und Kleinhirn verlorengegangen. 
hornformation sowie die Calcarina zeigen normale Verhältnisse. Im übrigen erscheint die graue Substanz sä mtlicher Großhirnwind ungen - allerdings in sehr verschieden erheblichem Maße - verbreitert, und zwar ist die Verbreiterung am stärksten an solchen Windungen, deren Oberfläche durch einen pachy gyren $^{\mathbf{1}}$ Typus, durch eine geringe Ausbildung der feinen tertiären Furchen auffällt (siehe Textfigur 1), entsprechend geringfügiger an Windungen mit annähernd normal

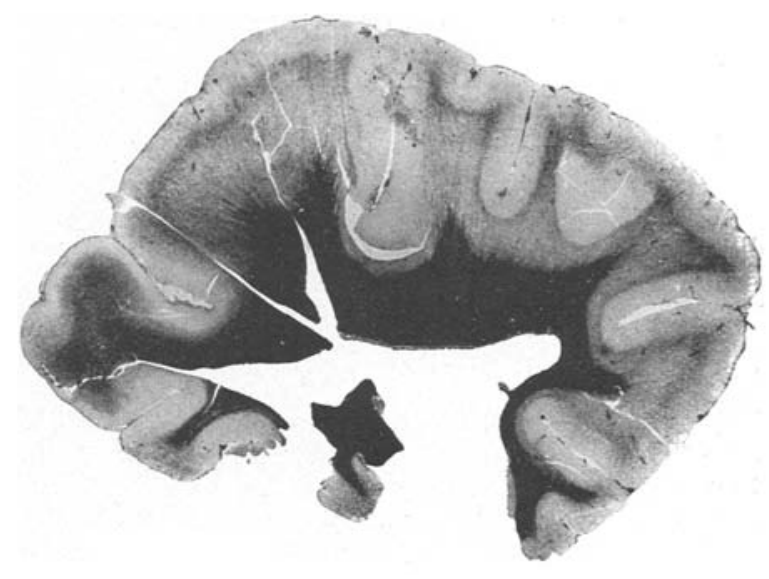

Fig. 1.

feiner Gliederung - wie z. B. im Stirnlappen (s. Textfigur 2).

Die schon am frischen Präparat beobachtete Zweiteilung dieser breiten Rinde ist an fast allen Windungen gut erkennbar. Die Grenze zwischen den beiden Rinden wird durch die Meynertschen U-Fasern (Fibrae arcuatae s. propriae) gebildet, die wir an gewohnter Stelle, d. h. in normalem Abstand von der Hirnoberfläche finden, so daß die oberflächliche Rinde etwa normale Breite zeigt. Die Grenze der in der Tiefe gelegenen Rinde gegen die eigentliche Marksubstanz ist überall unscharf und sehr unregelmäßig. Man findet daher hier im Schnitt Inseln von grauer Rinde durch Marksubstanz umschlossen; es handelt sich hier aber offenbar nicht etwa um Heterotopien, sondern einfach um Folgen der jeweiligen Schnittrichtung. Im übrigen ist die tiefe Rinde von wechselnder Breite, meist erheblich breiter als die oberflächliche. Es bleibt infolgedessen -

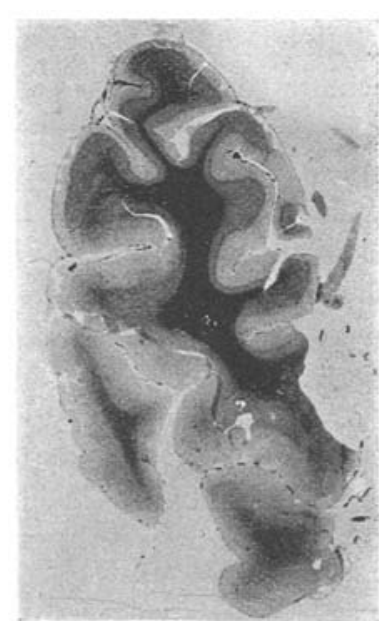

Fig. 2. an den Ventrikel sich anschließend - nur ein relativ schmaler Streifen geschlossener Marksubstanz übrig. Von diesem strahlen Markstrahlen

$\left.{ }^{1}\right)$ Ich gebrauche ebenso wie Ranke nach dem Vorschlag des Griechen Oeconomakis stets das Wort Pachygyrie statt Makrogyrie. 
aus, die den ganzen Rest der Hemisphärenbreite bis in die Windungskuppe hinein durchziehen, also gegenüber der Norm auf das Doppelte und mehr verlängert erscheinen. Es entsteht so das Bild einer radiären Streif ung eines großen Teils der Großhirnhemisphäre, die nur jeweils entsprechend den Furchentälern eine Unterbrechung erfährt.

Was die horizontalen Fasersysteme betrifft, so ist vor allem noch einmal die gute A usbild ung der k urzen, die einzelnen Windungen miteinander verbindenden Assoziationsfasern, also der Meynertschen U-Fasern, zu betonen. In der oberflächlichen Rinde findet man eine ziemlich breite, aber auffallend lockere Tangentialfaserschicht. Das supraradiäre Flechtwerk ist nur mäßig entwickelt, clas infraradiäre in normaler Stärke. In der tiefen Rinde findet man zwischen den kompakten Markstrahlen überall ein dichtes Netz von Markfasern, unter denen solche horizontaler Richtung überwiegen, ohne daß jedoch geschlossene Horizontalfaserbündel abzutrennen wären. Die langen Assoziationssysteme, so z. B. der Fasc. longit. infer. sind in normaler Weise angelegt. Uber die Commissurenfasern ist an dem sezierten Gehirn ein sicheres Urteil nicht abzugeben.

Gehen wir nun zur Betrachtung der Zellpräparate über, so finden wir zunächst sofort bestätigt, daß es sich bei der abnormen Bildung unterhalb der Fibrae arcuatae in der Tat um eine zweite Rindenanlage handelt: das ganze Gebiet ist erfüllt von Ganglienzellen. Von großem Interesse ist nun die Zellarchitektonik. Da dieselbe in allen Windungen - leider unterblieb eine Untersuchung der Zentralwindungen - im wesentlichen die gleiche ist, kann die Beschreibung die Befunde zusammenfassen. In der oberflächlichen Rinde finden wir in dem breiten Randsaum unmittelbar unter der Pia einige meist wohlausgebildete Cajalsche Horizontalzellen. Es folgt dann eine ziemlich breite ganglienzellenfreie Zone. In der Anordnung der eigentlichen Rindenganglienzellen fällt eine $\rightarrow$ bekanntlich gewissen Fötalperioden eigentümliche - besonders scharfe Ausrichtung in radiären Reihen vielfach auf, während die normalen sechs Meynertschen horizontalen Schichten meistens nicht zu identifizieren sind.

Während die Zellen der oberflächlichen Rinde, wie normal, überall senkrecht zur Oberfläche ausgerichtet sind, ist bei der tiefen Rinde augenscheinlich ein ganz anderes Prinzip maßgebend: hier streben die Ganglienzellen fast ausnahmslos der Kuppe der Windungen zu, ihre Achse steht stets parallel der Richtung der Fibrae arcuatae; sie steht daher nur unterhalb der Windungs $k$ uppe senkrecht zur Oberfläche, während sie unterhalb des Windungstals gerade parallel zur Oberfläche verläuft. Die Folge davon ist, daß wir, wenn wir einen Schnitt durch die Höh e einer Windung legen, sämtliche Ganglienzellen beider Rinden in langen, etwas auseinandergezogenen Reihen der 
Oberfläche zustreben sehen, wobei die Grenze zwischen normaler und pathologischer Rinde nicht erkennbar ist, daß wir dagegen auf einem durch ein Windungstal geführten Schnitt die Ganglienzellen an der Grenze zwischen oberflächlicher und tiefer Rinde plötzlich und scharf ihre Richtung ändern sehen, indem sie oberhalb dieser Grenze senkrecht zur Oberfläche, unterhalb dagegen parallel zu derselben orientiert sind (s. Tafel II, Fig. 1). Diese Grenze entspricht selbstverständlich dem Ort der Fibrae propriae. - Endlich ist noch zu erwähnen, daß auch in dem schmalen Rest eigentlicher Marksubstanz sich noch vereinzelte Ganglienzellen finden, etwa in der Anzahl, in der wir normalerweise die sog. Markzellen in den Markleisten finden. Dagegen finden sich in Ventrikelnähe weder restierende Keimbezirke noch ausgesprochene Heterotopien grauer Substanz.

Was die Art der Ganglienzellen betrifft, so ist hervorzuheben, daß es sich durchweg sowohl in der normalen wie in der tiefen Rinde um wohlausgebildete, reife Exemplare handelt. Nirgends findet man Zellen, die als unreife Neuroblasten oder gar als Körmerzellen zu bezeichnen wären. In den meisten Zellen läßt sich ein schönes intracelluläres Fibrillennetz nachweisen; wo das nicht der Fall ist, sind Mängel der Technik nicht auszuschließen, von zweikernigen Ganglienzellen habe ich trotz intensiven Suchens nur ein e inziges Exemplar gefunden. Häufiger sind zwei Nucleoli in einem Kern anzutreffen. Außerdem ist zu bemerken, daß man in der tiefen Rinde nicht selten zwei Zellen, mit kongruenten Flächen einander anliegend, dicht neben. einander findet. Dafür stehen aber die Zellen - resp. Zellpaare hier im ganzen weitläufiger als in einer normalen Rinde. Der Zwischenraum wird weniger durch Gliazellen ausgefüllt, als durch jenes von Nissl schlechtweg als ,Gra u“ bezeichnete Gewebe, dessen Masse durch die uns bekannten nervösen und gliösen Strukturen wohl noch nicht hinlänglich erklärt ist.

Gehen wir endlich zur Betrachtung der Stützsubstanz über, so finden wir den gliösen Randsaum überall deutlich verbreitert (s. Tafel II, Fig. 2). Von dem mäßig dichten oberflächlich gelegenen Netzwerk zweigen lange dünne Gliafasern ab, die, im wesentlichen sich dem normalen Grundriß des Gliagefüges in der Rinde einordnend, den ganzen zellfreien Raum erfüllen und bis in die äußerste Ganglienzellenlage hineinreichen; weiter in der Tiefe findet man, wie gewöhnlich, nur vereinzelte Gliafasern. Im Gebiete der zweiten pathologischen Rinde waren solche überhaupt nicht nachzuweisen. Die Gliazellen zeigen keine Abweichung von der Norm, speziell auch keine amöboide Umwandlung.

Am Gefäßsystem fällt eine ganz abnorm reichliche Entwicklung der corticalen Capillaren auf, welche am stärksten 
im Gebiete der pathologischen Rindenbildung ausgesprochen ist, wo ein ganz ungewöhnlich engmaschiges Capillarnetz anzutreffen ist, die aber auch in der oberflächlichen Rinde merklich über das Normale hinausgeht. Dabei weicht die histologische Struktur der Gefäße jedoch nicht von der gewöhnlichen ab. Gröbere Gefäße fehlen - wie auch beim normalen Gehirn - in der Rinde. Die der Pia zeigen in Bau, Anordnung und Anzahl nichts Auffallendes, abgesehen davon, daß natürlich - entsprechend der Pachygyrie - die in tiefe Furchen verlagerten Gefäße seltener sind. Auch das Grundgewebe der Pia läßt keine Anomalien erkennen, ebensowenig das Ependym der nicht erweiterten Seitenventrikel.

Wollen wir nun diese höchst eigenartige Mißbildung rubrizieren, so kann es keinem Zweifel unterliegen, daß man sie - ganz allgemein gesprochen - den Heterotopien grauer Substanz zuzurechnen hat. Daß es bei solchen zur Entstehung regelrechter windungsähnlicher Gebilde kommen kann, ist schon bei dem ersten überhaupt bekanntgewordenen Fall von Virchow beschrieben worden und auch durch weitere Fälle der Literatur geläufig (Meschede, Anton usw.). Es würde dann unser Fall nach von Monakows Einteilung der verschiedenen Heterotopiearten in die vierte Gruppe, nämlich die der subcorticalen Windungen, gehören, oder aber, wenn man H. Vogts Einteilung folgt, hätte man ihn dessen vierter Gruppe zuzurechnen, bei der die abgesprengten Teile typische Anordnung (z. B. Rindenformation, Olive usw.) zeigen.

Von den ausführlicher publizierten Fällen kommen dem meinen am nächsten die beiden von Meine und Matell mitgeteilten. Bei beiden handelt es sich um eine außerordentlich stark verbreiterte Rinde, die - wenn auch nicht überall - eine Zweiteilung erfährt durch die normale Ausbildung der Fibrae propriae. Aber beide Male handelt es sich um Gehirne, die - im Gegensatz zu dem meinen - auch anderweitig erhebliche Verbildung zeigen. So finden sich bei beiden Hetorotopien grauer Substanz auch am Ventrikel (bei Matell allerdings nicht mit absoluter Sicherheit festgestellt). Die Pachygyrie ist, wie ein Vergleich der Figuren sofort lehrt, viel erheblicher als in meinem Fall, namentlich bei dem von Matell beschriebenen Gehirn, dessen Oberfläche dort, wo die ,doppelte Rinde" vorhanden ist, überhaupt kaum irgendwelche Gliederung zeigt, so daß dann auch die die Zweiteilung bedingenden Fibrae propriae im Schnitt eine fast gerade Linie bilden; andererseits ist dort, wo eine normale Ausbildung der Furchen vorhanden ist, auch die Breite der Rinde eine normale. Vor allem aber ist der feinere A ufba $u$ der in der Tiefe gelegenen pathologischen Rinde in diesen Fällen ein ganz anderer: diese setzt sich, 
wie wir sahen, in unserem Falle aus wohlgereiften, nach einem ganz bestimmten Prinzip angeordneten Ganglienzellen zusammen; bei jenen dagegen handelt es sich neben vollentwickelten Ganglienzellen um viele unreife Neuroblasten und ganz indifferente Zellen, und diese sind nicht irgendwie ausgerichtet, sondern, wie Vogt für den Meineschen Fall ausdrücklich noch einmal nachgeprüft hat, in einer Grundsubstanz ganz unregelmäBig verteilt.

Der von mir beobachtete Fall zeigt demnach die hier in Frage kommende Anomalie nicht nur in sehr reiner Form - indem alle weitergehenden Mißbildungen vermißt werden - sondern auch in einer nach den bisherigen Erfahrungen ungewöhnlichen Höhe der Differenzierung. Denn wie die genannten Fälle von Meine und Matell, verhalten sich in dieser Beziehung alle übrigen irgendwie ähnlich gelagerten Fälle, sowie die nicht so sehr wesensverschiedenen Fälle von Mikrogyrie ${ }^{1}$ ) (siehe Anton, Oeconomakis, Kotschetkowa, von Monakow, Liebscher u. a.). Oeconomakis vergleicht solche Heterotopien mit einem im Stich gelassenen Neubau, dessen Baumaterial umherliegt, und Vogt gibt an, daß Störungen in der Reifung der Elemente nie fehlen, wo solche der Wanderung und der Gruppierung vorhanden sind. Von dieser Regel macht dann unser Fall eine Ausnahme, denn Störungen der Wanderung müssen wir, wie wir gleich sehen werden, doch wohl sicher hier annehmen.

Von sonstigen Fällen der Literatur kann, als entfernte Ähnlichkeit zeigend, noch der erste der Vogtschen Fälle genannt werden, bei dem unter einer sehr wenig gegliederten Oberfläche sich eine verschieden stark verbreiterte, sehr unregelmäßig gegen das Mark abgegrenzte Rinde befand - auch hier mit fehlender Ausrichtung der in der Entwicklung zum Teil stark zurückgebliebenen Zellelemente. Doch fehlt hier ebenso wie in dem von Probst veröffentlichten Fall von ,Makrogyrie" der in den anderen Fällen so charakteristische Markstreifen an der Grenze zwischen oberflächlicher und tiefer Rinde. Das gleiche gilt für den Fall Koch der Marchandschen Publikation, den Meine mit zu dieser Gruppe rechnet ${ }^{2}$ ).

Wenn wir nunmehr den Versuch machen, uns ein Bild von der Entstehung einer solchen Mißbildung zu entwerfen, so müssen wir vorweg

1) Ich spreche hier und im folgenden trotz der Ausführungen Rankes, der die meisten dieser Fälle zum Status corticis verrucosus (simplex oder deformis) rechnet, stets von Mikrogyrie, da dieser Name hier zunächst nur rein mor phologisch gebraucht wird, überdies aber auch nach Bielschowsky genetisch zutreffend ist.

2) Dagegen wäre hier ein Gehirn zu erwähnen, das Alzhei mer auf der Breslauer Neurologentagung demonstriert, aber, soweit ich sehe, nicht durch den Druck publiziert hat, bei dem sogar eine dreifache Rindenanlage vorhanden war. 
die Frage erörtern, ob es sich bei dem pathologischen Rindengewebe in der Tiefe um überschüssiges Zellmaterial oder nur um am unrechten Ort befindliches handelt, m. a. W., ob wir eine Mißbildung per excessum vor uns haben, oder eine solche per defectum. Diese Frage kann nicht ohne weiteres beantwortet werden, denn die Abschätzung, ob die Gesamtzahl der Ganglienzellen die normale übersteigt oder nicht, ist unmöglich. Sie wäre nur dann mit Sicherheit im Sinne einer Exzessivbildung zu beantworten, wenn man annehmen könnte, daß schon die oberflächliche Rinde allein annähernd die normale Ganglienzellenzahl enthielte. Das ist aber sicher nicht der Fall, denn an der mangelhaft gegliederten pachygyren Oberfläche findet - bei etwa normaler Breite der oberflächlichen Rinde und sicher nicht abnorm enger Zellanordnung - natürlich lange nicht die gleiche Anzahl von Zellen Platz, wie in einem Gehirn mit gut ausgeprägter Furchenbildung. Ob dieses Defizit durch die Zellmassen in der Tiefe gedeckt, nicht gedeckt oder überkompensiert wird, das zu entscheiden ist unmöglich. Einen besonderen Anhalt für eine abnorm reichliche Zellproduktion haben wir jedenfalls nicht. Ein solcher würde z. B. vorliegen, wenn wir - was ja aber nicht der Fall ist - noch jetzt etwa vasculäre oder ventrikuläre Keimbezirke ( $\mathrm{R}$ an ke), womöglich gar an ungewohnter Stelle fänden, die auf eine über die normale Zeit hinaus dauernde übermäßig starke Zellproliferation hinweisen würden. So glaube ich, daß wir die Annahme einer Exzessivbildung, die Ranke bei der Mikrogyrie resp. dem Status corticis verrucosus deformis unumgänglich erscheint, in unserem Fall wohl entbehren können.

Eine zweite Frage ist die, ob wir es bei dieser zweiten Rindenbildung nicht vielleicht mit einer qua si nor malen Erschein ung zu tun haben, d. h. mit einer solchen, die beim Foetus regelrechterweise und auch im extrauterinen Leben nur in quantitativ geringerem Maße zu beobachten ist. Ranke hält die zahlreichen Nervenelemente, die man bis in den siebenten Fötalmonat hinein namentlich an der Markrindengrenze findet (,Nervenzellen des Markes"), nicht wie His für wandernde Neuroblasten, sondern, da er sie schon zu Zeiten, in denen die Rindenpyramidenzellen noch völlig unreif sind, kräftige Fortsätze ausbilden sah, für Nervenzellelemente sui generis, die ähnlich wie die Cajalschen Horizontalzellen im wesentlichen nur der Fötalzeit angehören und schon vor deren Abschluß regressive Veränderungen eingehen. Er glaubt, daß die reichlichen Markzellen, die man z. B. in Idiotengehimen und bei juveniler Paralyse findet (Ranke, Rondoni), und die nach H. Vogt in Gehirnen mit Entwicklungsstörungen überhaupt nie vermißt werden, solche Fötalzellen darstellen, bei denen pathologischerweise die sonst eintretende Rückbildung ausgeblieben ist. Ich möchte hierzu bemerken, daß die Zahl der ja in jedem Gehirn 
anzutreffenden Markzellen individuell in außerordentlich hohem Grade variiert und da $\beta$ man sie auch in solchen Gehimen, die sonst keinerlei Zeichen von Entwicklungsstörung bieten, bisweilen in ganz überraschend großer Menge findet. Ohne nun die von Ranke gegebene Deutung für solche und ähnliche Fälle anzuzweifeln, möchte ich sie für meinen Fall durchaus ablehnen. Hier haben wir sicher etwas ganz anderes vor uns als persistierende Fötalzellen. Der Unterschied ist keineswegs nur ein quantitativer, denn wir haben es hier eben nicht mit ungewöhnlich vielen Ganglienzellen im Mark zu tun, sondern mit einer richtigen Rindenbildung. Dafür ist maßgebend, abgesehen von der vielfach erwähnten Ausrichtung der Elemente, die Eigenart der $Z$ wischensubstanz, die zwar - bis auf den Mangel resp. die Seltenheit von Gliafasern - noch schwer zu definieren ist, die aber doch ein von dem des Marks deutlich abweichendes ganz charakteristisches Gepräge hat.

Einen sehr eigenartigen Erklärungsversuch für die uns hier beschäftigende Mißbildung macht Matell: Er glaubt, daß das Primäre eine Minderentwicklung des Marks darstellt und daß die Ganglienzellen der tiefen Rinde nur Füll material für die so entstehende Lücke im Aufbau der Hemisphärenwand darstellen. Ich glaube kaum, daß diese Vorstellung berechtigt ist. Wir haben allen Grund anzunehmen, daß bei mangelhafter Anlage der Marksubstanz das vorhandene Ganglienzellmaterial sich den veränderten Verhältnissen in der Weise anpassen würde, daß es zur Bildung mikrogyrer Windungen kommt. Denn wenn auch diese - namentlich von Heschl, Anton, Zingerle, Liebscher gegebene - Erklärung der Mikrogyrie heute auf Grund der Arbeiten von Chiari, Probst, Oeconomakis, Groz, Ranke, Bielschowsky $u$. a. als allgemeingültige zumeist verlassen ist, so kann man doch auch jetzt noch dem Moment der primären Markverbildung für gewisse Fälle von Mikrogyrie eine gewisse Rolle zusprechen. Das sind ja nun aber ganz andere Erscheinungsformen als wir sie in unserem Falle vor uns haben. Auch erscheint die Vorstellung, daß das wachsende Gehirn als Ersatz für nicht zur Entwicklung gekommene Marksubstanz Ganglienzellen bildet, zum mindesten völlig unbewiesen.

Wollen wir dem Verständnis einer solchen Mißbildung näher kommen, so müssen wir natürlich von den normalen Vorgängen bei der Entwicklung der Großhirnrinde ausgehen. Die Kenntnis dieser Dinge, die übrigens von Vollständigkeit noch weit entfernt ist, verdanken wir vor allen Dingen $\mathrm{H}$ is und weiterhin neben vielen anderen $\mathrm{Ranke.}$ Danach findet sich die Brutstätte der Ganglienzellen in den in Ventrikelnähe gelegenen Keimbezirken. Von hier aus wandern unreife Neuroblasten aus, durchqueren die ganze Breite der Hemi- 
sphäre und sammeln sich unweit der Oberfläche an. Hier ordnen sie sich unter stetiger Weitervermehrung nach gewissen Umlagerungen zu den uns bekannten Schichten und reifen zu ausgesprochenen Nervenzellindividuen aus. Wenn wir uns an diesen Entwicklungsgang halten, so kommen wir ganz von selbst dazu, die bei uns vorliegende Anomalie so zu deuten, wie auch sonst die Entstehung von Heterotopien erklärt wird, nämlich dadurch, daß ,in frühester Fötalzeit gewisse cerebrale Einzelanlagen an der für eine spätere erfolgreiche architektonische Ausgestaltung notwendigen Ortsveränderung (und Einstellung ihrer Elemente in typischen Gruppen) verhindert werden" (v. Monakow). Nehmen wir an, daß aus irgendeinem Grunde die Wanderung der Neuroblasten ganz generell verzögert wird, so müssen zweifellos gerade die Verhältnisse eintreten, wie wir sie in unserem Falle finden. Besonders möchte ich hier auf die Rolle hinweisen, die die Fibrae propriae der Rinde für die Konfiguration dieser doppelten Rindenanlage spielen: sie sind offénbar, unbeeinflußt durch die Anomalien der Anordnung der Ganglienzellen, am normalen Ort - ob auch zur normalen Zeit, muß unentschieden bleiben - angelegt worden ${ }^{\mathbf{1}}$ ), und durch diese horizontale Barriere werden die verspätet eintreffenden Neuroblasten definitiv an dem Vordringen bis zu dem ihnen in der Norm bestimmten Platz gehindert. Für ein derartiges ,Abgeschnittenwerden" von Zellen durch Fasern haben wir ein physiologisches Beispiel im Linsenkern, der nach $\mathrm{His}$ als ,ein Stück abortiv gebliebene Rinden-

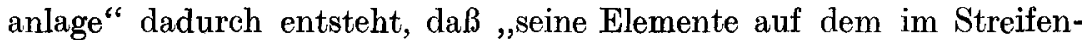
hügelgebiet besonders langen Weg zur Peripherie stehengeblieben und durch weiße Fasermassen umgrenzt worden sind". Ebenso müssen sich in unserem Falle die Nervenzellen unterhalb der U-Fasern angesammelt haben, und werden, wenn ihnen die normale Wachstumstendenz innewohnte, hier zu reifen Nervenzellen ausgewachsen sein. Es hat sich dann die entsprechende Zwischensubstanz gebildet, und durch Ordnung in bestimmten Richtungen ist es zu dem Bild einer wahren Rindenformation gekommen. Interessant ist dabei, daß für diese besondere Anordnung der Elemente offenbar die Fibrae propriae ma:Bgebend gewesen sind.

Andererseits ist nun mit dem verzögerten Ausschwärmen n ur ein Teil der Neuroblasten bis an die Oberfläche gelangt. Nun ist es nach

1) Rondoni gibt gerade in bezug auf die Meynertschen U-Fasern an, daß „die Zellen immer der Festpunkt, der Hauptbestandteil, das Charakteristicum der Rinde sind und die Lage der Fasern bestimmen". Unser Fall spricht gegen diese Auffassung, man müßte denn die nicht sehr wahrscheinliche Annahme machen, daß zwischen der Bildung der beiden Rinden ein Stadium existiert hätte, in welchem der jetzt von der zweiten Rinde eingenommene Raum ganz frei von Ganglienzellen gewesen wäre. 
der sehr plausiblen Theorie Rankes ,die an differenten Orten verschieden lebhafte Proliferation des Keimmaterials, welcho auch im Laufe der normalen Rindenfurchung die Änderungen der äußeren Gestalt verursacht". Es kann daher einerseits, wie Ranke an Hand der Befunde der Züricher Schule ausführt, nach dem Gesetz der Selbstdifferenzierung auch in der Tiefe zurückgebliebenes Bildungsmaterial höherer grauer Zentren den vorgeschriebenen Billungsgang innehalten $-d$. h. also $\mathrm{z}$. B. auch sich zu richtigen Windungen umbilden - , andererseits aber muß, wo die Zellproliferation an der Oberfläche erheblich hinter dem Normalen zurïckbleibt, ein wesentlicher Anla $B$ zur Furchenbildung fehlen: letztere wird dana, wie in unserem Falle, in engeren Grenzen bleiben als normal, der Windungstypus wird pachygyr werden.

Man könnte sich ja nun allerdings denken, daß es sich gerade u mgekehrt verhielte, daß das Primäre eine abnorm geringe Furchung wäre und daß so unter der zu kleinen Oberfläche nicht alle Ganglienzellen Platz gefunden hätten und deshalb in der Tiefe liegengeblieben wären. Aber abgesehen davon, daß die eben genannte, sehr plausible Theorie der Ursache der Furchenbildung damit nicht vereinbar wäre, scheint mir angesichts des sehr deutlichen Einflusses, den die Fibrae propriae offenbar auf die Lagerung der Zellelemente ausgeübt haben, sowie des Umstandes, da $\beta$ die feineren sekundären und tertiären Windungen, um die es sich hier handelt, erst zu einer Zeit angelegt werden, in der die Wanderung der Neuroblasten in der Hauptsache vollendet sein soll, die erste Möglichkeit die bei weitem wahr scheinlichere zu sein. Ohne strikte beweisbar zu sein, erklärt diese Annahme m. E. in jeder Beziehung am besten die vorliegenden Verhältnisse.

Aber wie dem auch sei, jedenfalls ist hervorzuheben die enge Beziehung, die zwischen der Furchenbildung und der Anordnung der Ganglienzellen besteht: wir haben gesehen, daß in unserem Fall, je ausgesprochener die zweite Rindenbildung, desto deutlicher die Pachygyrie über dieser Stelle ist; wir bemerkten in dem Fall von Matell, daß n u r unter der fast ungegliederten Oberfläche die Anomalie der Rinde zu erkennen war, an den Windungen mit normaler Furchung jedoch fehlte. Es vermag das wohl einen gewissen Ausblick zu geben auf die Verhältnisse bei der normalen Hemisphärenentwickl ung, wie ja überhaupt die teratologischen Befunde ein nicht zu entbehrendes Hilfsmittel der embryologischen Forschung clarstellen (Vogt). Bekannt ist die Deutung, die Anton ${ }^{1}$ ) der Furchenbildung gegeben hat.

1) Diese Deutung wird in der Literatur fast stets mit dem Namen Je lg e r s mas verknüpft. Sie ist aber bereits 4 Jahre vor der Mitteilung dieses Autors in klar formulierter Weise von Anton, der sich dabei auf Maudsley stützt, gegeben worden. (Zeitschr. f. Heilk. \%, 454. 1886.) 
Er erinnert daran, daß, wenn die Hemisphäre nach allen drei Di mensionen im $K u b u s$ wächst, die zweidimensionale Oberfläche nur im Quadrat zunimmt. Wenn daher die an der Oberfläche situierte Rinde mit dem Wachstum des Marks Schritt halten soll, so muß, falls sie nicht immer dicker werden soll, eine Fältelung eintreten. Dieses „falls sie nicht immer dicker werden soll" hat im allgemeinen nur wenig Beachtung gefunden. Man hat einfach als Faktum hingenommen, daß jeder Tierspezies eine bestimmte Hirnrindenbreite zukommt, und daß auf dem Wege der Verdickung ein Wachstum der Rinde nicht möglich ist.

Hierfür haben nun schon Reichert und Meynert eine Erklärung in der Gefäßverteilung zu finden gesucht: sie machen darauf aufmerksam, daß grö bere Gef ä ße in der Hirnrinde durchweg ver mieden sind, daß vielmehr die Speisung des Rindencapillarnetzes von den Gefäßen der Pia aus erfolgt. Soll diese für die äußerst sauerstoff- und nahrungsbedürftige Rindensubstanz ausreichen, so darf die Entfernung der zu versorgenden Gebiete von den Pialarterien nicht zu groß, m. a. W. die Rinde nicht zu breit sein. Ist dies schon für das ausgewachsene Gehirn unerläßlich, so ist es von doppelter Bedeutung für die in Entwicklung begriffene Rinde, da sich an die Gefäße die Vegetationszentren der Ganglienzellvermehrung anschließen (Ranke).

Interessant sind nun die Belege, die uns für diese Anschauung teratologische Befunde von zwei verschiedenen Seiten her bieten: Hier haben wir einerseits die Fälle von Mikrogyrie, bei der abnorm zahlreiche und abnorm seichte Furchen gebildet werden. Für ihre Entstehung macht schon Ranke abnorme Gefäßverhältnisse verantwortlich, und Bielschowsky sieht das Wesentliche in einem „Zusammenwirken der veränderten Vascularisation, welche mit der Entwicklung atypisch lokalisierter Vegetationszentren auf das engste verknüpft ist, und der verminderten Evolutionstendenz der zelligen Elemente in diesen Zentren". Hierdurch wird ein erhebliches Defizit an Rindenzellenmaterial bedingt, das nur in unvollkommener Weise dadurch kompensiert wird, daß ,an zahlreichen Gefäßen zwischen den ursprünglichen Vegetationsstreifen neue Keimzentren sich entwickeln", welche - in die Tiefe gelangend - zur Bildung meist nur seichter neuer Furchen Anlaß geben.

Andrerseits zeigen die Fälle von Meine, Matell und mir, daß ausnahmsweise die Volumzunahme der Rinde doch einmal auf dem Wege des Dickenwachstums vor sich gehen kann, indem die Ganglienzellen nur zum Teil bis in die Nähe der Oberfläche gelangen, und daß in solchen Fällen dann die Furchenbild ung hinter der Norm entsprechend weit $\mathrm{z}$ urückbleibt${ }^{1}$ ).

1) Diese Bildungen scheinen mir eher dem ,,von Reichert geahnten, Gehirn, 
Wenn die oben mitgeteilten Ansichten richtig sind, so ist zu erwarten, daß in einem solchen Falle dann auch die Gefäßversorgung eine at y pische sein wird. Das ist nun in der Tat der Fall, allerdings nicht in dem Sinne, daß etwa abnormerweise gröbere Gefäße in die Hirnsubstanz selbst verlagert wären, wohl aber so, daß sich im Gebiet der pathologischen Rinde ein ganz abnorm dichtes Capillarnetz findet, das anscheinend sowohl von der Pia wie vom Ventrikel her gespeist wird. (Etwas Ähnliches ist in dem Fall von Matell vermerkt, sowie in einigen anderen, wie z. B. dem von Probst.) Damit soll nun keineswegs etwa behauptet werden, daß diese Gefäßanomalie das Primäre ist. Nur soviel kann man sagen, daß auf Grund obiger Anschauungen eine solche pathologische Rindenbildung ohne gleichzeitige Anomalien in der Gefäßanordnung nicht wohl bestehen kann.

Man würde somit folgende Gegenüberstellung machen können: Bei der Mikrogyrie zeigen die in normaler Weise bis zur Oberflächennähe gelangten Ganglienzellen eine abnorm geringe, aber doch an verschiedenen Stellen verschieden erhebliche Vermehrungstendenz, wodurch die Tiefe der Furchen reduziert wird bei gleichzeitig erfolgender ungenügender Kompensation durch deren abnorme Zahl. Bei der Pachygyrie von unserm Typus kommt dagegen ein beträchtlicher Teil der Zellen dadurch, daß er der Oberfläche fernbleibt, überha u pt nicht dazu, Einfluß auf deren Gestaltung zu gewinnen. Für die übrigbleibenden ist auch an einer wenig gegliederten Oberfläche genügend Platz; die Furchenbildung wird sowohl an Zahl, wie auch an Tiefe hinter der Norm zurückstehen. In beiden Fällen aber wird man a ußerdem eine abnorme Gefäßanordnung als wesentlich für das Zustandekommen dieser Mißbildungen annehmen müssen. Daß die Entwicklungshemmung bei den Fällen von Pachygyrie in einem früheren Stadium eingesetzt haben muß, als in denen von Mikrogyrie, ist nach obigem klar.

Was nun den eigentlichen Anla $ß$ gegeben hat zu der angenommenen Verzögerung im Ausschwärmen der Neuroblasten, das zu entscheiden scheint mir unmöglich, wie uns denn überhaupt in den meisten Fällen solcher Mißbildungen „das primäre pathologische Moment“" (Vogt) verborgen bleibt. Nur soviel kann man sagen, daß kein Anhalt für die Annahme einer entzündlichen Genese vorliegt, nach der man in solchen Fällen so oft forscht und für die man gelegentlich in

bei dem die Windungen fehlen und an Raum gespart sein würde“, zu entsprechen, als der von Ranke herangezogene Status corticis verrucosus deformis. Denn bei diesem ,senken sich“, wie der genannte Autor selbst ausführt, „mit dem Streifen des Molekularsaums besonders weite blutstrotzende Gefäße in die Tiefe". Es liegen hier also eigentlich quoad Gefäßversorgung genau die gleichen Verhältnisse vor, wie bei normaler Furchenbildung. 
Verwachsungen, Beziehungen zu den Gefäßen. und dgl. eine gewisse Stütze findet (Oppenheim, Kalischer, Ranke u. a.), und daß der Zeitpunkt, in der die Störung eingesetzt haben muß, zwischen dem 4. und 6. Fötalmonat zu suchen ist, in welcher Zeit die Abgrenzung zwischen Rinde und Mark vor sich geht und jedenfalls vor dem Stadium anzunehmen ist, in welchem die Meynertschen U-Fasern zur Anlage kommen ${ }^{1}$ ), wodurch die subcorticale Lage dieser Rindenteile definitiv fixiert wird.

Will man sich endlich die Beziehungen zwischen pathologisch-anatomischem Befund und klinischem Symptomenbild vergegenwärtigen, so sind hier hauptsächlich $\mathrm{z}$ wei Fragen von Interesse; einmal: Inwieweit sind mit einer solchen pathologischen Großhirnentwicklung normale cerebrale Funktionen vereinbar? Zweitens: Besteht ein Zusammenhang zwischen dieser Anomalie und den epileptischen Anfällen, und welcher Art ist er?

Die erste Frage ist an der Hand unseres Falles schwer zu beantworten. Ich habe schon oben betont, daß nach Einsetzen gehäufter epileptischer Anfälle eine Weiterentwicklung nicht mehr zu erwarten ist und wir daher bei unserer Patientin nur die Zeit bis zum Alter von $5 / 4$ Jahren berücksichtigen können. Daß für die Störungen auf somatischem Gebiet die Rachitis sehr wesentlich maßgebend ist, haben wir ebenfalls schon gesehen. Aber auch nach Abzug der hierauf zurückzuführenden Symptome bleibt eine gewisse Minderentwicklung, z. B. auf dem Gebiet der Sprache. Dieselbe ist aber insofern als nicht hochgradig zu bezeichnen, als die Sinnesfunktionen offenbar normal entwickelt waren. Jedenfalls erscheint der Allgemeinstatus im Alter von 5/4 Jahren nicht als ein solcher, daß eine annähernd der Norm entsprechende Entwicklung für die Zukunft vollständig ausgeschlossen gewesen wäre. $\mathrm{Ob}$ diese gegebenenfalls stattgefunden haben würde, ist natürlich unmöglich $\mathrm{zu}$ entscheiden. Ziehen wir die anatomisch ähnlichen Fälle von Meine und Matell zu Rate, so finden wir, daß hier beide Male recht erh ebliche geistige Defekte vorgelegen haben. Dabei ist aber zu berücksichtigen,' daß in beiden Fällen, wie mehrfach hervorgehoben, die Entwicklungsstörungen viel ausgebreiteter und viel weitergehend waren als in dem meinen, so daß man aus jenen nicht ohne weiteres Rückschlüsse auf diesen ziehen kann.

Dagegen läßt die weitere Tatsache, daß in diesen drei Fällen ebenso wie in sehr vielen der mit ihnen entfernter verwandten übereinstimmend epileptische Anfälle zur Beobachtung kamen, die Frage nach dem eventuellen Zusammenhang zwischen diesem Symptom und der Entwicklungsstörung des Gehirns dahin entscheiden, daß es sich

1) Diese Fasern werden, wie viele andere, erst nach der Geburt markhaltig. Ihre eigentliche Anlage fällt natürlich in einen viel früheren Zeitpunkt. 
jedenfalls nicht um ein zufälliges Zusammentreffen handeln dürfte. Wie der Zusammenhang zu denken ist, das soll erst nach Mitteilung des zweiten Falles gemeinschaftlich mit diesem erörtert werden.

Da bei diesem zweiten, in der Hallenser Nervenklinik beobachteten Fall, den mir Herr Geh. Rat Anton gütigst zur pathologischen Untersuchung überließ, der klinische Verla uf von besonderem Interesse ist, so mag hier die Krankengeschichte etwas ausführlicher wiedergegeben werden.

Lina J., Bergmannskind. Bei der Aufnahme 12 Jahre alt. Stammt aus vollkommen gesunder Familie und hat sich bis zum 10. Lebensjahr in völlig normaler Weise entwickelt. Sje war in der Schule sogar eine der Besten. Dann stellten sich e pile ptische Anfälle ein, die allmählich an Zahl und Stärke zunahmen und zur Zeit der klinischen Aufnahme fast täglich ein- bis fünfmal auftraten. Von Petit-Mal-Anfällen war anamnestisch nichts zu eruieren. Seit Beginn der Krämpfe war allmählich ein sehr weitgehender geistiger Verfall aufgetreten. Menstruiert war Pat. noch nicht.

Bei der objektiven Untersuchung am 16. I. 1911 hat man ein im Wachstum vielleicht um ein geringes zurückgebliebenes, aber sonst körperlich seinem Alter entsprechend entwickeltes Kind vor sich. Der Hirnschädel ist symmetrisch, der Gesichtsschädel dagegen zuungunsten der rechten Hälfte etwas uns y m metrisch. Dabei ist die Facialisinnervation beiderseits normal.

Es besteht ganz leichter Exophthalmus. Der Augenhintergrund ist beiderseits völlig intakt. Die Pupillenreaktion zeigt keine Besonderheiten. Der Blick ist unstet, vorzugsweise nach rechts gerichtet, doch sind die Augenbewegungen nach allen Richtungen ausgiebig möglich. Corneal- und Conjunctivalreflex erhalten. An der Zunge einige Narben. Zähne auffallend verstellt. Die Gaumen. platte steil.

Die Sehnenreflexe sind wenig lebhaft, kein Babinski. Von den Bauchdeckenreflexen sind nur die beiden oberen und der linke mittlere auslösbar. Die übrigen Hautreflexe zeigen nichts Pathologisches, ebensowenig, soweit zu prüfen, die Motilität und Sensibilität. Beim Gehen dreht Pat. den Kopf meist nach der rechten Schulter, das Kinn nach links, im übrigen ist der Gang ungestört.

An den inneren Organen ist außer leichter Pulsbeschleunigung nichts Abnormes festzustellen. Die Trommelfelle zeigen narbige Veränderungen. Sonst Ohrbefund ohne Besonderheiten. Wassermannreaktion negativ. Hämoglobin $75 \%$. Gefärbtes Blutpräparat ohne Besonderheiten.

In psychischer Beziehung ist folgendes festzustellen: Der Gesichtsausdruck ist stumpf und apathisch, die Mimik teilnahmlos. Spontanbewegungen führt Pat. nur äußerst spärlich aus. Sie zeigt kein Interesse für die Vorgänge im Saal. Ebenso fehlt es so gut wie ganz an s pontanen sprachlichen Äußerungen. Nur zuweilen ruft sie „Papa, Papa“. Es scheint, als ob sie Gegenstände mehr beachtet, wenn sie von der rechten Seite kommen, sie faßt aber nur selten - dann stets mit der rechten Hand - nach ihnen. Auch Abwehrbewegungen werden meist mit der rechten Hand ausgeführt. Einf achste verbale Aufforderungen führt sie nicht aus. Es läßt sich auch sonst kein Anhalt dafür gewinnen, daß Pat. Verständnis für das gesprochene Wort hat. Überhaupt fehlt es an jeder Aufmerksamkeit für akustische Eindrücke. Demgegenüber macht sie einfache, ihr vorgeführte Bewegungen, wie Zungeherausstrecken, Armehochheben, prompt nach. 
Pat. hat gleich bei der Aufnahme und in der Folgezeit zwei- bis dreimal täg. lich A nfälle von $1 / 2$ bis 2 Minuten Dauer mit Bewußtlosigkeit, Enuresis, ziemlich schwachen klonischen Krämpfen in Armen und Beinen. Kopf und Augen sind dabei bald nach rechts, bald nach links gewendet, die Pupillen maximal erweitert und lichtstarr.

Bei einer Nachuntersuchung 4 Tage später ist zum ersten Male eine Reaktion auf Sprachliches zu konstatieren: sie zeigt die $Z$ unge nach mehrf a cher Wieder holung der Aufforderung, perseveriert hieran aber bei dem Befehl, die rechte Hand ans rechte Ohr zu legen. Auf die Frage: „Wo wohnst du ?" erfolgt unter starkem Unmutaffekt nach längerer Zeit: „Ich weiß nicht." Aber auch jetzt noch erweist sich das Wortverständuis als erheblich herabgesetzt. Objekte bezeichnet sie nicht. Der Gebrauch von Trompete und Mundharmonika erfolgt richtig, ausgeschüttete Bohnen liest sie zusammen und tut sie in die Schach. tel, zum Anzünden eines Streichholzes ist sie nicht zu bewegen. Das Kind muß an- und ausgezogen und aus dem Bett genommen werden.

Weitere vier Tage später ist das Kind, nachdem auf Brommedikation hin die Anfälle schwächer geworden sind, auffällig munterer, es zeigt Interesse für seine Umgebung und lebhaftere Affektäußerungen, freut sich beim Besuch des Vaters, weint, als er fortgeht. Es spricht jetzt eine Reihe von vorgesprochenen Wörtern nach, zeigt aber für verbale Aufforderungen so gut wie kein Verständnis. Dagegen geht sic - was sehr bemerkenswert ist - auf Gestensprache ein. Einzelne Gegenstände benutzt sie richtig, z. B. Trompete, gebraucht dann aber perseverierend Zahnbürste und Kamm wie Trompete, sagt dazu: „Weeß nicht." Ihr vorgemachte Bewegungen imitiert sie, wenn auch bisweilen nicht ganz korrekt und dann wieder an der vorigen Aufgabe klebend. Der Wortschatz ist äußerst dürftig geblieben. Auf Diktat und Vorschrift schreibt sie statt ihres Namens (Julitz): ,i, i, Jiizi.“ Einige Zahlen schreibt sie nach, auch hierbei tritt Perseveration ein.

Bei der kleinen Patientin wurde wiederholt folgender Versuch gemacht:

Sie reagierte nicht auf Worte, unternahm auch keinerlei Kopfdrehung nach dem Anredenden zu. Dagegen war sie für Rhythmus empfänglich, und bei den Tanzstücken eines größeren Grammophons hat sie sich zeitweilig zum Tanzen an. geregt gefühlt und dabei richtig Rhythmus und Schritt gehalten. Zu solchen Zeiten gelang es auch, die meist stupide Pat. in heitere Stimmung zu versetzen.

Bezüglich des Kopf-Röntgenbildes sei noch nachgetragen, daß der Schädel mit Ausnahme des Hinterhauptteiles relativ dünn war.

Die Gegend des Clivus war nach abwärts etwas ausgewölbt und verstrichen, die Nebenhöhlen des Schädels ohne besonderen Befund.

20. II.: Körperliches Befinden und psychisches Verhalten schwanken in nicht sehr weiten Grenzen meist parallel der Häufigkeit der Anfälle. Heute löst sie leichte Rechenaufgaben wie $3+2,3 \times 3$, versagt aber bei Subtraktionen.

10. III.: Pat. malt Kreise und Kreuze nach, auch mit der linken Hand, schreibt aber auf Aufforder ung nichts weiter als ihren Namen, in den sie auch bei anderen vorgesprochenen Wörtern zurückfällt. Nachschreiben gelingt bei Wörtern wie Papa, Mama, nein, Halle, lateinisch deutlich ungeschickter als deutsch. Sie schreibt, nachdem ihr 1 vorgeschrieben ist, die Zahlen bis 26 richtig.

27. III. : Heute nach einer Serie von 3 schweren Anfällen leichte Reflexstei gerung und Verminderung der groben Kraft links gegenüber rechts. Kein Babinski. Auch im übrigen neurologischer Befund negativ. Augenhintergrund und Pupillen dauernd ohne Besonderheiten.

28. III.: Die Reflexdifferenz ist wieder verschwunden. 
Auch im April wechseln entsprechend der Anfallhäufigkeit Zeiten schlechteren Befindens, in denen Pat. außerordentlich stumpf, fast benommen ist, mit solchen, in denen sie heiter ist, singt und sich nach ihrer Art spielend beschäftigt.

28. IV.: Die deutsch vorgeschriebenen Wörter Ida: liest sie „da", Papa: „Pa“", Mama: "Ma“. Sie schreibt auf Diktat ihren Namen und Papa richtig, statt Mama „Mullutti“, statt Halle „Mäll“.

Am .2. V. wird auf der chirurgischen Klinik eine Punktion des linken Schläfenlappens vorgenommen. Die histologische Untersuchung des gewonnenen Hirnstückchens ergibt keinen pathologischen Befund.

Am 2. und 30. Mai tritt ein schwerer Stat us e pilepticus auf, in dessen Gefolge sich eine Schluckpneunomie entwickelt.

Bei der Zurückverlegung in die Nervenklinik am 2. VI. ist Pat. äußerst verfallen, es erfolgen leichte Unruhbewegungen der Hände. Pat. reagiert auf akustische und Schmerzreize mit leichtem Blinzeln. Im körperlichen Status keine Veränderung.

Am 13. VI.: Exitus letalis.

Wir haben hier also einen Fall von Epilepsie mit häufigen Anfällen vor uns, bei dem es ungewöhnlich schnell zu einer schweren geistigen Dekadenz gekommen ist. Dabei mögen allerdings die Symptome der letzteren infolge der fast stets vorhandenen, wenn auch an Intensität wechselnden Trübung des Bewußtseins noch schwerwiegender erschienen sein, als dem tatsächlichen geistigen Besitzstand entsprach. Jedenfalls ist aber an dem Bestehen eines erheblichen Defektes nicht zu zweifeln.

Analysiert man denselben aber genauer, so findet man, daß es sich keineswegs um eine gleichmäßige Einbuße aller intellektuellen Fähigkeiten handelt, sondern daß manche Störungen - und zwar solche, die wir zumeist bei herdförmigen Hirnerkrankungen zu finden gewohnt sind - derartig in den Vordergrund treten, daß man ihnen wohl eine selbständige Stellung einräumen muß. Mag das für die meist durch Perseveration bedingten Fehlreaktionen auf dem Gebiet des Erkennens und Handelns fraglich erscheinen, so trifft es doch sicher zu für die fast vollkommenem Verlust gleichkommende Herabsetzung des Sprachverständnisses. Eine richtige Reaktion auf verbale Aufforderungen ist überhaupt nur zweimal notiert: einmal wird der Befehl, die Zunge zu zeigen, nach mehrfacher Wiederholung befolgt, das andere Mal handelt es sich auffälligerweise um das Verständnis für leichte Rechenaufgaben. Wenn Pat. dagegen auf die Frage: „Wo wohnst du?" mit ,Ich weiß nicht" antwortet, so ist dafür nur die schon durch den Tonfall zu gewinnende - Auffassung der Frage als solcher, nicht aber ein Verständnis ihres Inhaltes nötig.

Auf motorischem Gebiet ist eine schwere Verarmung des Wortschatzes im allgemeinen zu konstatieren. Die spärlichen zur Verfügung stehenden Wörter werden aber sinngemäß angewandt, korrekt, nicht paraphasisch ausgesprochen. Ob man die Fehler der schriftlichen Wortwiedergabe in das Gebiet der Paragraphie rechnen 
soll, mag dahingestellt bleiben; man muß berücksichtigen, daß die Kunst des Schreibens bei einer 10jährigen noch nicht allzu fest sitzt, und daß da nach über zweijähriger schwerer Krankheit nicht mehr allzuviel zu erwarten ist. Andererseits spricht die Art der Fehler „Mulutti“ für Mama, „Mäll“ für Halle - bei richtiger Ausführung der einzelnen Schriftzeichen doch in gewissem Grade für paragraphische Störungen, wobei dann nur das Fehlen von Paraphasien auffallend wäre.

Es kann keinem Zweifel unterliegen, daß die beobachteten Erscheinungen allgemein gesprochen ins Gebiet der aphasischen gehören. Die beiden sonst allein noch in Betracht kommenden Ursachen der mangelhaften Reaktion auf sprachliche Reize sind auszuschließen: Schwerhörigkeit schon angesichts des leidlich guten Nachsprechens. Der Allgemeinzustand des Bewußtseins und der Aufmerksa $m$ keit ist allerdings wohl geeignet, derartige Störungen zu begünstigen, und auf den meist der Häufigkeit der Anfälle parallel gehenden Wechsel auf diesem Gebiet sind wohl die Schwankungen im Nireau des Sprachverständnisses zurückzuführen. Aber die einzige oder auch nur hauptsächliche Ursache kann auch hierin nicht gefunden werden, wenn man ihr Verhalten bei dem erwähnten Grammophonversuche bedenkt und berücksichtigt, wieviel prompter yorge mach te Bewegungen von ihr imitiert als mündliche Aufträge ausgeführt werden und daß sie auch auf Gestensprache eingeht. Es bleibt also nur die Annahme übrig. daß hier eine Worttaubheit - im weiteren Sinne vorliegt. Die Einordnung in eine bestimmte Gruppe der Aphasie dürfte jedoch recht schwer fallen, da die Symptomatologie zu keinem der bekannten Typen passen will. Um eine reine subcorticale Wortta ubheit kann es sich nicht handeln, da das Nachsprechen erhalten ist --, zur eigentlichen sensorischen A phasie - sei es der corticalen, sei es der transcorticalen - kann das Krankheitsbild aber auch nicht gerechnet werden, da nicht nur im Gegensatz zu der diesen Gruppen sonst eigenen Logorrhöe eine große Verarmung auf motorischem Sprachgebiet vorhanden war, sondern vor allem auch $\mathrm{Paraph}$ asien, wie es scheint, fehlten. Man wird demnach nur von einer starken Reduk. tion des Sprachverständnisses sprechen dürfen, ohne diese Erscheinung näher zu klassifizieren.

Jedenfalls aber legte der klinische Befund es nahe, an eine Herderkrankung im Bereich des linken Schläfenlappens zu denken, und es war zweifellos berechtigt, den Versuch zu machen, ob sich durch Probepunktion etwa ein operables Leiden feststellen ließe. Das Resultat war jedoch negativ. Und ebenso negativ blieb zunächst das Ergebnis der Sektion: äußerlich war weder am linken Schläfenlappen noch sonst irgendwo eine auffällige Veränderung festzustellen 
und auch nach Härtung und Zerlegung in Frontalschnitte war ein positiver Befund nicht zu erheben. Dagegen vermochte die mikroskopische Untersuchung wenn auch den Fall nicht völlig zu klären, so doch einige interessante Ergebnisse zutage zu fördern.

Zunächst fällt bei Markscheidenpräparaten von Frontalschnitten eine durch das ganze Gehirn verstreute Lichtung der Markfasern auf, die sich streng an das Gebiet der Projektionsfasern hält und in den beiden Schläfenlappen noch etwas ausgesprochener ist als an den anderen Stellen (s. Textfigur 3). Es treten dadurch die Fasern anderer Dignität in ganz auffallender Weise hervor, wie z. B. auf der Fig. an dem Fasciculus longitudinalis inferior deutlich ist. Dem Markscheidenbild entspricht das Biclschowsky-Präparat, welches eben-

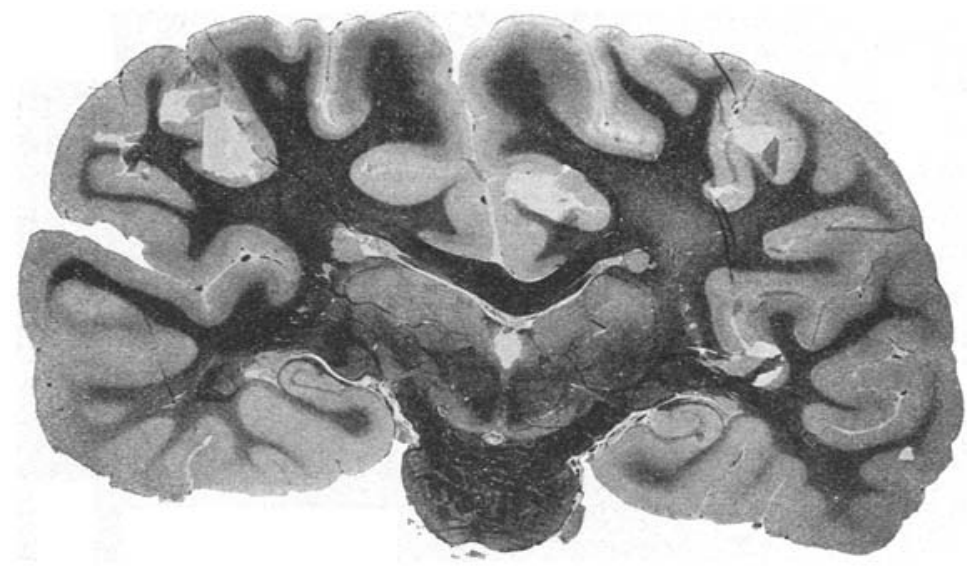

Fig. 3.

falls hier deutlich eine weniger dichte Anordnung der Nervenfasern erkennen läßt. Irgendein dieser Faserverarmung zugrundeliegender pathologischer Prozeß konnte durch die histologische Untersuchung nicht festgestellt werden: Weder findet man Abbauzellen, noch eine Reaktion von seiten der Glia, die darauf hinweisen würde, daß hier Nervensubstanz zugrunde gegangen ist. Dieses negative Ergebnis im Zusammenhang mit der Elektivität, mit der überall allein die Projektionsfasern betroffen sind, läßt wohl keine andere Deutung zu als die, daß es sich um eine von Haus a us mangelhafte Anlage handelt.

Als ein weiterer an der ganzen Hirnoberfläche nachweisbarer Befund ist die Persistenz zahlreicher Cajalscher Horizontalzellen zu nennen, die nur zum Teil erhebliche Anzeichen von Degeneration zeigen, zum Teil aber wohlerhalten sind.

z. f. d. g. Neur. u. Psych: O. XXXIII. 
Drittens findet sich überall am Großhirn der gliöse Randsa um deutlich mehr oder weniger erheblich verbreitert und verdichtet (s. Taf. III, Fig. 3): die langen, ziemlich feinen Fasern bilden ein dichtes Netzwerk und reichen stellenweise auch ziemlich weit in die Tiefe, fügen sich aber in die normale Architektonik gut ein. Am stärksten ist diese Randgliose wohl am linken Schläfenlappen ausgesprochen, außerdem sind die einzelnen Fasern hier bedeutend dicker (s. Tafel III, Fig. 4). In diesem Himabschnitt fand sich nun des weiteren in der Marksubstanz unweit der Rinde ein kleiner Herd von etwa Bohnengröße, der sich histologisch als ein kleines ziemlich scharf begrenztes Gliom erwies. Da dieser Herd erst beim Schneiden des entsprechenden Blocks bemerkt wurde, so ist leider eine genauere Lokalisierung desselben versäumt worden ${ }^{1}$ ).

Damit ist erschöpft, was an abweichenden anatomischen Befunden im Gehirn des Kindes J. zu erheben war. Wenn ich nunmehr eine Deutung des Zusammenhanges zwischen klinischen und pathologischen Erscheinungen versuche, so will ich von vornherein erklären, daß ich eine sichere Entscheidung, welche von den beiden hier in Betracht kommenden Möglichkeiten die zutreffende ist, nicht zu geben vermag. Man könnte sich natürlich vorstellen, daß es sich in unserem Fall um einen primären gliomatösen Hirntumor im linken Schläfenlappen handle, der als cerebrale Allgemeinerscheinungen die (sc. symptomatischen) epileptiformen Anfälle sowie einen Teil der psychischen Symptome hervorgerufen hätte. Für diese Ansicht könnte man vor allem die $\mathrm{Hä} \mathrm{ufigkeit} \mathrm{epileptischer}$ Anfälle gerade bei Schläfenlappentumoren anführen — nach Astwazaturow 22 Mal unter 43 Fällen - sowie den Umstand, daß in solchen Fällen die Krämpfe den übrigen Symptomen jahrelang vorausgehen, ja endgültig das einzige Symptom bleiben können (Astwazaturow). Als Herderscheinung wäre dann die Störung des Sprachverständnisses aufzufassen, wobei man allerdings nur an eine Fernwirkung denken könnte; denn es erscheint mir ausgeschlossen, daß dieser kleine Herd, wenn auch seine Entfernung von der Wernickeschen Stelle nicht genau bestimmt werden konnte, eine genügende Anzahl von Fasern getroffen haben sollte, um einen so erheblichen Defekt als reine Ausfallserscheinung zu bedingen.

Aber diese ganze Auffassung, wenn sie sich auch nicht widerlegen läßt, scheint mir doch etwas Gezwungenes zu haben. Das Mißverhältnis zwischen Ursache und Wirkung wäre doch etwas

1) Die Ammonshorngegend stand mir leider zu einer genaueren histologischen Erforschung nicht zur Verfügung. Auf Markscheidenpräparaten erschien das Ammonshorn der einen Seite schmäler als das der anderen, ohne daß ich über links und rechts eine Entscheidung fällen könnte. 
z u groß. Gewiß besteht kein Parallelismus zwischen der Größe einer Geschwulst und den durch sie hervorgerufenen Symptomen. Wir wissen aber auch, daß, wenn kleine Tumoren schwere Erscheinungen machen, dies zumeist durch Vermittlung eines vermehrten Hirndrucks geschieht und daß es sich deshalb in solchen Fällen meist um Neubildungen in der hinteren Schädelgrube handelt, während die Tu moren an anderen Stellen oft sehr groß werden können, ehe sie irgendwelche Allgemeinerscheinungen hervorrufen. Nun haben aber im Falle J., wie ausdrücklich betont werden muß, alle Zeichen vermehrten Hirndruckes gefehlt: Weder war intra vitam irgendein darauf hindeutendes Symptom wie Staungspapille, Erbrechen usw. nachzuweisen, noch wies bei der Obduktion irgendein Befund - wie etwa Abplattung der Windungen, Hydrocephalus oder dgl. - auf das Vorhandensein eines pathologischen Hirndrucks hin. Bei dieser Sachlage scheint mir eine zweite Auffassung des Falles mehr Befriedigung zu gewähren: Man könnte sich nämlich sehr wohl vorstellen, daß es sich um eine echte, oder wenn wir den Ausdruck gebrauchen wollen, genuine-jedenfalls nicht nur symptomatische - E pile psie handelt und daß die anatomischen Gehirnveränderungen auf diese Erkrankung resp. auf eine ihr zugrundeliegende Entwicklungsstörung des Gehirns zu beziehen sind. Auf diese Weise könnte man dieselben auch sehr wohl unter einheitlichem Gesichtspunkt betrachten, während bei Annahme eines primären Tumors einige Veränderungen als zufällige Nebenbefunde hingehen müßten.

Hierher gehört vor allem die Lichtung im Gebiet der Projektionsfasern, die, wie ich bei Schilderung des histologischen Befundes auseinandergesetzt habe, wohl sicher als auf von vornherein mangelnder Anlage beruhend angesehen werden muß.

Auch der weitere Befund einer Persistenz Cajalscher Horizontalzellen wird allgemein als ein Symptom einer Entwicklungshemmung angesehen. Nach Ranke sollen sie normalerweise bei der Geburt bis auf geringe Reste verschwunden sein, und ihr Weiterbestehen in späteren Lebensjahren soll ein u. a. gerade der genuinen Epilepsie eigenes pathologisches Vorkommnis sein. Nun habe ich allerdings gar nicht selten typische und wohlerhaltene Exemplare dieser Zellen, gerade so, wie ich das oben von den "Markzellen" berichtet habe, bei der Sektion Erwachsener an einer beliebigen somatischen Erkrankung verstorbener Individuen in Gehirnen, die sonst durchaus keinen Anhalt für eine mangelhafte Entwicklung boten, gefunden. Auch Jakob erwähnt ihr Vorkommen bei Arteriosklerose und seniler Demenz, also in Fällen, bei denen wir a priori eine kongenitale Entwicklungsanomalie anzunehmen keinen Anlaß haben. Ob, wie Jakob angibt, quantitative Unterschiede in dem Sinne bestehen, daß die Zellen bei 
Epileptikern in viel reichlicherer Menge angetroffen werden als in anderen Fällen, das zu entscheiden fehlt mir das genügende Material an Epileptikersektionen. In den erwähnten Fällen von Nichtepileptischen waren sie zum Teil gar nicht einmal sehr spärlich vorhanden. Immerhin kann man vielleicht sagen, daß das Restieren von Zellen, die wohl in der Mehrzahl der Fälle einer physiologischen Degeneration schon von Geburt an verfallen, auf eine gewisse Minderentwicklung hindeutet.

In dritter Linie ist hier das kleine Gliom zu nennen. Bekanntlich wird diese Tumorart vielfach ebenfalls mit kongenital mangelhaften Differenzierungsvorgängen in cer Glia in Verbindung gebracht, und wenn auch, wie Redlich hervorhebt, eine feste Begründung dieser Anschauung noch fehlt, so läßt sich doch mancherlei zu ihren Gunsten anführen ${ }^{1}$ ).

Was endlich die Randgliose betrifft, so könnte diese Eracheinung eventuell auch von dem Gliom abhängig gemacht werden. Denn nach den neuen Untersuchungen von Stern kommt eine Verstärkung des gliösen Randsaums bei Hirntumoren in vereinzelten Fällen ebenfalls vor und scheint - nach den Figuren zu schließen - ausnahmsweise auch ähnliche Grade erreichen zu können, wie in unserem Falle, ohne daß sichere qualitative Unterschiede gegenüber der für die Epilepsie so charakteristischen Veränderung beständen. Aber immerhin stellt dies Vorkommnis doch eine A usnahme dar und ist auch von Stern nur in Fällen beschrieben, bei denen ein voluminöser Tumor mit sonstigen cerebralen Allgemeinerscheinungen vorhanden war. Und so scheint es mir auch hier ungezwungener, diesen Befund mit der Erkrankung in Verbindung zu bringen, bei der er in einem so hohen Prozentsatz der Fälle erhoben wird, der Epilepsie. Bekanntlich wird im Gegensatz zu der älteren von Chaslin vertretenen Auffassung nach den Untersuchungen Alzheimers diese Randgliose nicht etwa als Ursache der Erkrankung, sondern als Folge des bei jedem einzelnen Anfall zustandekommenden und sich so allmählich summierenden Ausfalls an Nervensubstanz betrachtet. Mit Recht aber macht Jakob darauf aufmerksam, daß man auch hier vielleicht eine durch $D$ ifferenzierungsstörungen bedingte Tendenz der Glia zu abnorm starker Reaktion zur Erklärung zu Hilfe nehmen muß, da diese Reaktion weit über diejenige hinausgeht, die z. B. in Fällen seniler Demenz bei viel erheblicherer Rindenatrophie zur Beobachtung kommt. Dieser Anschauung schließt auch Bielschowsky sich für gewisse Fälle an auf Grund der Untersuchungsergebnisse eines Falles, in dem die Anomalien der Glia allerdings sowohl quantitativ wie auch quali-

1) Neuerdings hat Bielsch owsky neue gewichtige Gründe für diese Annahme beigebracht. 
tativ von der gewöhnlichen Chaslinschen Gliose ziemlich erheblich abweichen, und der bezeichnenderweise mit einem diffusen Ponsgliom kombiniert war.

Endlich könnte man außerhalb des Gehirns noch die A nomalien i m Schädelbau: die Asymmetrie des Gesichtsschädels, den steilen Gaumen mit Verstellung der Zähne als Stigmata gestörter Entwicklung anführen.

Die zweite mögliche Auffassung unseres Falles J. ginge demnach dahin, daß, wie im ersten Fall, auch hier das Primäre eine Entwicklungsstörung des Zentralorgans darstellt, welche in den verschiedenen erwähnten pathologisch-anatomischen Abweichungen von der Norm, eventuell auch in dem Gliom, ihren Ausdruck findet, und daß auf diesem Boden sich die Krankheit Epilepsie entwickelt hat, als deren Folgezustand wir die Randgliose anzusehen hätten. Der Umstand, daß die Verdickung des gliösen Randsaums im lin ke n Schläfenlappen am stärksten a usgesprochen ist, auch an Stellen, die ziemlich weit von dem Tumor entfernt sind, brauchte dann nicht einfach als Reaktion auf die Neubildung gedeutet zu werden, eine Auffassung, die sehr erschwert wird durch die Tatsache, daß die eigentliche Gliareaktion in der Umgebung des ja in der Marksubstanz gelegenen Tumors ziemlich unbedeutend und schon in geringer Entfernung von demselben nicht mehr erkennbar ist; vielmehr würde dieser Befund dann nur als ein Hinweis darauf anzusehen sein, daß der Prozeß seinen Ausgang vermutlich von dem Hirnabschnitt genommen hat, der durch die Gegenwart des Glioms sich als ein besonderer locus minoris resistentiae dokumentiert.

Dementsprechend würde man dann auch die ,a phasischen Störungen" nicht als direktes Tumorsymptom aufzufassen haben, sondern man würde sie den schon seit langer Zeit bekannten und namentlich in jüngster Zeit seit Redlichs Untersuchungen besonders gewürdigten Herderscheinungen bei Epileptikern einreihen müssen. Wenn man im allgemeinen bei den hier in Betracht kommenden Symptomen postparoxysmale und intervalläre zu unterscheiden pflegt, so kann, wie auch Heilbronner auseinandersetzt, in Fällen mit gehäuften Anfällen, wie der unsere einer ist, diese Unterscheidung nicht durchgeführt werden. Praktisch haben wir dann jedenfalls Dauersymptome vor uns. Solche Dauersymptome pflegen ja nun in der Regel nur in unscheinbaren Abweichungen von der Norm, Reflexdifferenzen $u$. dgl. zu bestehen (Redlich); daß aber auch a usgesprochene Hemiparesen bisweilen dauernd vorhanden sein können, dafür gibt es mehrere Beispiele in der Literatur, u. a. den Fall von Stransky. Was nun speziell die a phasischen Erscheinungen betrifft, so scheinen nach den Untersuchungen von Heilbronner 
gerade bei ihnen UUbergänge vorzukommen zwischen den relativ häufigen passageren Erscheinungen im Anschluß an Anfälle und einem chronischen Defekt auf diesem Gebiet. Bei der pathogenetischen Deutung solcher Befunde kann ich mich ganz auf Heilbronner beziehen. Dieser Autor nimmt an, daß ,,auch als Grundlage der aphasischen Erscheinungen im Rahmen der Epilepsie ein lokalisierter Prozeß, zum mindesten auf bestimmte Stellen beschränkte stärkere pathologisch-anatomische Veränderung angenommen werden darf und bei hinreichend genauer Untersuchung auch nachgewiesen werden kann." In der Regel handelt es sich nach seinen Erfahrungen hierbei allerdings um Störungen amnestisch-aphasischer Art, die für eine Lokalisierung besonders ungünstig sind. Immerhin kann man sagen, daß dies Symptom mit der sensorischen Aphasie verwandt ist und im Sprachreflexbogen vor der Broca schen Stelle liegt. Genau das gleiche trifft auf die im Fall J. vorliegende, auch nicht genau zu lokalisierende Störung auf dem Gebiet des Sprachverständnisses zu. Wenn wir nun den anatomischen Prozeß der Randgliose im linken Schläfenlappen, der doch für den sensorischen Anteil dér Sprache in erster Linie in Frage kommt, besonders stark ausgesprochen finden, so scheinen die Heilbronnerschen Anschaungen durch unsern Fall volle Bestätigung zu erfahren, wenn auch $z$ ugegeben werden muß, daß die Gegenwart des kleinen Glioms die Bedeutung dieses Befundes etwas einzuschränken geeignet ist.

Die vorgetragene Auffassung der Dinge stimmt im wesentlichen überein mit derjenigen, die Steiner für seinen Fall vorgebracht hat ${ }^{1}$ ). Nun haben die Steinerschen Ausfïhrungen eine verschiedene Beurteilung erfahren: Ja kob, der bei dem zweiten der von ihm mitgeteilten Fälle diffuse, möglicherweise auch auf Entwicklungsstörungen zu beziehende Veränderungen an der Glia fand, zitiert sie in zustimmendem Sinne. Astwazaturow, der, wie erwähnt, sich mit dem häufigen Zusammentreffen von Schläfenlappentumoren und Epilepsie beschäftigt, glaubt eine Bestätigung der Anschauung Steiners darin zu finden, daß unter den mit Epilepsie einhergehenden Schläfenlappenneubildungen a uffallend hä ufig Gliome vertreten sind. Im übrigen spricht er über den Zusammenhang zwischen den beiden Erkrankungen keine bestimmte Ansicht aus; er weist nur auf die Nähe des A m mons horns und die dadurch nahegelegten zwei Möglichkeiten hin, daß entweder Schläfenlappentumoren durch ihre Wirkung auf das benachbarte Cornu ammonis epileptische $\mathrm{K}_{\mathrm{I}}$ ämpfe zur Folge haben oder um-

1) Es dürfte nicht ganz bedeutungslos sein, mitzuteilen, daß ich mir den oben als vorzugsweise wahrscheinlich dargestellten Zusammenhang der Erscheinungen zurechtgelegt hatte, bevor mir S teiners Arbeit bekannt war. Als die Publikation von Bielschowsky erschien, war die vorliegende Arbeit bereits druckfertig. 
gekehrt bei Epileptikern die Ammonshorngegend ein locus minoris resistentiae ist und dadurch günstige Bedingungen für die Entwicklung einer Neubildung bietet. Endlich hat Bielschowsky sich für seinen vorher erwähnten Fall mit Ponsgliom ganz die Stei nersche Anschauung zu eigen gemacht, wobei er auf die nahe Verwandtschaft zwischen blastomatösen Prozessen und Mißbildungen hinweist.

Demgegenüber lehnt Redlich die Steinersche Auffassung strikte ab: Die von Astwazaturow eruierte Häufigkeit der Gliome in solchen Fällen entspricht nach seinen Erfahrungen dem Prozentsatz dieser Geschwulstart unter den Hirntumoren überhaupt. Daß die Randgliose wie Steiner annimmt, nicht zu den bei Hirntumoren nachwejsbaren diffusen Veränderungen gehöre, sei bei der Spärlichkeit diesbezüglicher Untersuchungen vorerst noch nicht als feststehend $\mathrm{zu}$ erachten. Er weist darauf hin, daß Reichardt bei Hirntumoren von Verbreiterung des Gliasaums und oberflächlicher Gliose spricht (in demselben Sinne die oben erwähnten Untersuchungen Sterns). Redlich nimmt in solchen Fällen an, daß wenn nicht ein zufälliges Nebeneinandervorkommen vorliegt, die epileptischen Anfälle Folge der Hirngeschwulst sind.

Auch ich muß zugeben, daß Steiner einen Beweis für seine Anschauung nicht erbracht hat. Ich habe in meinem Falle eine sichere Entscheidung, welche Auffassung die für ihn zutreffende sei, nicht zu geben vermocht und habe nur die zweite, der Steinerschen entsprechende, für diejenige gehalten, die die größere Wahrscheinlichkeit für sich hat. In meinem Falle spricht aber im Gegensatz zu dem von Steiner sehr zugunsten dieser Anschauung neben manchen vorher erwähnten Einzelheiten, ganz besonders das a ußerordentliche Mißverhältnis, das zwischen Ursache und Wirkung bestehen würde, wenn man alle Erscheinungen allein auf das bohnengroße Gliom zurïckführen wollte.

Endlich möchte ich noch eine dritte Möglichkeit, die zwischen den beiden bisher erörterten vermittelt, wenigstens namhaft machen. Man könnte sich nämlich natürlich auch vorstellen, daß zwar Entwicklungsstörungen, wie sie in den verschiedenen pathologischen Befunden zum Ausdruck kommen, das Primäre darstellen, daß aber in letzter Linie es doch der Hirntumor ist, der auf dem so vorbereiteten Boden - und nur auf solchem - zur Entstehung der epileptischen Anfälle geführt hat.

Den beiden mitgeteilten Fällen, so verschieden sie sowohl im klinischen Verlauf als auch im pathologisch-anatomischen Befund sich uns darstellen, ist das ge meinsa $\mathrm{m}$, daß beide Male einem epileptischen Leiden intra vitam der Sektionsbefund von Entwicklungsstörungen 
des Zentralorgans entspricht. Wir müssen deshalb zum Schluß noch kurz in eine Erörterung darüber eintreten, wie diese Koinzidenz z u bewerten ist, insbesondere, ob diese anatomische Anomalie als direkte Ursache der Erkrankung an Epilepsie zu betrachten ist ${ }^{1}$ ). Diese Frage ist natürlich nicht ohne weiteres $\mathrm{zu}$ beantworten, weil wir über die eigentliche Ursache dieser Krankheit nichts wissen. Die reine Empirie lehrt uns aber, daß - abgesehen von den einmalige epileptische Krämpfe hervorrufenden Ereignissen - u. a. chronische Vergiftungen, Residuen von Traumen und Entzündungen, Hirndruck steigernde Prozesse die Veranlassung für die Entstehung eines epileptischen Leidens geben können, wenn auch der Mechanismus im einzelnen noch unbekannt oder wenigstens strittig ist. $\mathrm{Ob}$ aber die Entwicklungsstörungen des Gehirns als solche die Ursache der Epilepsie darstellen, darüber wissen wir nichts. Wahrscheinlich erscheint mir dies keineswegs. Denn erstens wäre es dann schwer zu erklären, warum das Leiden nicht schon von Geburt an besteht (Beginn bei meinem ersten Fall im Alter von 11/4, beim zweiten im Alter von $10 \mathrm{Jah}-$ ren), da es sich doch weder um sich summierende Schädlichkeiten, wie etwa beim Alkoholismus, noch um einen allmählich zunehmenden Proze $\beta$ handelt, wie bei Tumoren und Hydrocephalus, noch endlich um einen solchen, der sekundär andere Gebiete des Gehirns in Mitleidenschaft zieht, wie Traumen und Entzündungen.

Zweitens aber führt von diesen relativ groben und eingreifenden Störungen im Aufbau des Gehirns eine ununterbrochene Reihe bis zu jenen, anscheinend geringfügigen, zum Teil auch ganz circumscripten Anomalien, denen ganz allgemein eine ätiologische Bedeutung nicht zugeschrieben wird. Wo will man nun da die Grenzo setzen? Will man die Verlagerung eines großen Teils der grauen Hirnrinde wie in meinem Fall I verantwortlich machen für die Entstehung der Epilepsie, einer vereinzelten Heterotopie in Ventrikelnähe dagegen jede ätiologische Bedeutung absprechen? Entschieden müssen diese Fälle unter einheitlichem Gesichtspunkt betrachtet werden. Und dieser einheitliche Gesichtspunkt kann nur der sein, daß die min derwertige Entwicklung des Gehirns, auf die solche Anomalien hinweisen, den günstigen Boden abgibt für die Entstehung der Krankheit Epilepsie, nicht aber deren direkte Ursache ist. Die Epilepsie ist nicht abhängig von den speziellen Abnormitäten, sondern beide sind koordinierte Symptome der mangelhaften Hirnanlage - wenn man es kraß ausdrücken will, Stig mata degenerationis.

Ist diese Arsschauung richtig, so liegt kein Anlaß vor, Fälle wie die

1) Die folgenden Ausführungen treffen auf den zweiten Fall natürlich nur unter der Voraussetzung zu, daß die „zweite Möglichkeit“ die richtige ist. 
hier mitgeteilten anders zu beurteilen als andere, und für den, der nicht mit Redlich den Begriff der genuinen Epilepsie ganz fallen lassen will, besteht kein Grund, von dieser Gruppe u n sere Fälle auszuschließen. Denn so viele verschiedene Definitionen der, ,ge n u in en " Epilepsie auch heute noch existieren, auf die zwei mitgeteilten Fälle treffen sie doch alle zu: Gehen wir zunächst von einem pathologisch-a nat o m ischen Einteilungsprinzip aus, so wird heute wohl niemand mehr der ,,genuinen" Epilepsie als „,organische“ oder ,,symptomatische" alle diejenigen Fälle gegenüberstellen wollen, bei denen die makroskopische und mikroskopische Untersuchung des Gehirns überhaupt irgendwelche Veränderungen aufdeckt. Denn abgesehen davon, daß dann die Gruppe der genuinen Epilepsie, wie eingangs dieser Arbeit auseinandergesetzt, schon jetzt fast verschwunden sein würde, hieße das doch zum Maßstab der Einteilung die menschliche Unkenntnis und Unfähigkeit machen, einen glücklicherweise großen und schnellen Veränderungen unterliegenden Faktor.

Vielmehr wird es nötig sein, hier die in der Einleitung von mir versuchte Einteilung der pathologischen Befunde nach ihrer prinzipiellen Bedeutung zu berücksichtigen; dann ergibt sich von selbst, daß man als Fälle „organischer Epilepsie, “ wenn anders dieser Begriff überhaupt irgendeinen Sinn haben soll, nur solche bezeichnen darf, bei denen sich orga nische Veränderungen aus der ersten der dort angeführten Gruppen finden, d. h. solche, die man als eigentliche Ursache der Erkrankung ansprechen kann. Natürlich ist auch diese Einteilung aus denselben Gründen anfechtbar. Man kann aber soviel sagen, da $\beta$ diejenige Epilepsieform, die man auch aus anderen Gründen als genuine abzutrennen noch einen gewissen Anhalt hat, heutzutage in diesem Sinne als organische noch keineswegs gelten kann. Es soll hier nicht auf diese Streitfrage näher eingegangen werden. Es sei nur kurz darauf hingewiesen, daß gerade die konstantesten Befunde, wie die A m monshornsklerose und die Alzheimersche Gliose nach heutigen Anschauungen keineswegs als die Ursache der Erkrankung angesehen werden können, und daß selbst Redlich zugibt, daß wir zwar „eine", aber nicht „die" pathologische Anatomie der Epilepsie besitzen.

Bei Anwendung dieser Utberlegungen auf unsere Fälle ergibt sich auf Grund der vorhergehenden Erörterungen, daß, so in die Augen springend auch die vorhandenen Anomalien sind, doch bei ihnen eine als Ursache des epileptischen Leidens anzusprechende pathologische Veränderung nicht aufzufinden ist, daß sie demnach im pathologischanatomischen Sinne als genuine anzusprechen sind.

Ebenso dürfte eine große Anzahl ähnlich liegender Fälle aus der Literatur zu beurteilen sein. Ich denke hier z. B. an die Fälle von 
Balkenmangel, bei denen Epilepsie so häufig vorkommt; und bei denen wohl ganz allgemein der fehlende Balken nicht als die direkte Ursache des epileptischen Leidens angesehen wird (s. Landsbergen). Und so scheint mir auch die Sonderstellung, die z. B. Jakob seinen Fällen wegen der bei ihnen erhobenen speziellen Befunde trotz ihrer sonstigen Ähnlichkeit mit der gewöhnlichen chronischen Epilepsie gibt - zum mindesten für seinen zweiten Fall -, nicht zu Recht zu bestehen. Man kann aus solchen Fällen nur lernen, wie verschiedenartig diese Stigmata gestörter Entwicklung sein können. Was die tuberöse Sklerose betrifft, so möchte ich mich hier eines Urteils enthalten, zumal die Streitfrage, ob blastomatöser Prozeß oder reine Entwicklungshemmung, noch nicht entschieden ist.

Sehen wir nun des weiteren von der anatomischen Einteilung ab und halten uns an die ätiologische, die unter dem Namen der genuinen Epilepsie alle diejenigen Fälle zusammenfaßt, die - bei sonst dunkler Ätiologie - auf einer angeborenen, eventuell hereditären, Anlage beruhen sollen, oder sprechen wir mit Binswanger von einer dynamisch-konstitutionellen Form der Epilepsie, so leuchtet ohne weiteres ein, daß man dann erst recht unsere Fälle dieser Gruppe einreihen muß. Daß einer besonderen, a nor malen Konstitution bei der "genuinen" Epilepsie eine große Rolle zukommt, darüber herrscht wohl keine Differenz. Man spricht von einer „e pileptischen Veranlagung“. Es kann nicht ausgeschlossen werden, daß bei ihr konstitutionelle Anomalien des Stoffwechsels und der inneren Sekretion mitspielen, aber sicher kommt hier in erster Linie die besondere „Reaktionsfähigkeit" des Gehirns in Frage. die auf der besonderen - sich so häufig als von der Norm abweichend erweisenden - Anlage des Zentralorgans beruht. So und ähnlich urteilen Jakob und viele andere, und Binswanger gibt an, daß die dynamischen Vorgänge, als welche sich uns die Krankheitserscheinungen der echten Epilepsie darstellen, neben Keimschädigungen ,,auf angeborenen, ererbten, in der neuropsychischen Konstitution des erkrankten Individuums gelegenen Bedingungen beruhen" und daß "diese Konstitutionsanomalie sich in einem Teil der Fälle auch morphologisch kundgibt, bald in gröberen, schon makroskopisch erkennbaren Entwicklungshemmungen des Gehirns, ... bald in feineren nur mikroskopisch erkennbaren Hypoplasien". Die Konsequenz, die sich aus solchen Anschauungen m. E. zwingend ergibt, ist die, daß a uch die Fälle mit groben Entwicklungshem mungenzurgenuinen Epilepsie zu rechnen sind.

Wie Redlich auseinandersetzt, kommen, ebenso wie für andere Nerven- und Geisteskrankheiten, auch für die Epilepsie nebeneinander prädisponierende und auslösende, also endogene und 
e xogene Momente genetisch in Betracht, und oft besteht eine gewisse Gegensätzlich keit zwischen beiden, insofern die einen um so geringfügiger sein können, je schwerwiegender die anderen sind. Man könnte also nach diesem Gesichtspunkte die Epilepsiefälle in eine Reihe ordnen, und nun ist klar, daß wenn wir eine genuine Epilepsie als angeborene resp. konstitutionell-dynamische anerkennen, wir sie an dem Ende der Reihe zu suchen haben, wo die endogenen Momente weit über die exogenen überwiegen. Hier finden aber sicher auch unsere Fälle Platz: nicht nur, weil die Anomalie der Anlage bei ihnen so in die Augen springend ist, sondern weil die Träger solcher Anomalie - wie aus der Literatur wenigstens für die Källe vom Typus meines ersten, zu ersehen ist, — quasi a uf jeden Fall, gleichgültig was für innere und äußere Bedingungen sonst auf sie einwirken, epileptisch werden. Möglicherweise sind für solche Gehirne schon die normalen Schwankungen des Stoffwechsels ein zur Auslösung von Krampfanfällen genügender Reiz. Man könnte daher wohl mit Recht fragen: Wenn es überhaupt eine genuine Epilepsie gibt, wo soll man sie suchen, wenn man diese Fälle nicht hierherrechnen will?

Fälle wie die unseren weisen zwar besonders eindringlich auf die Bedeutung solcher Entwicklungsstörungen für die Pathogenese der Epilepsie hin. Aber daß sie qualitativ nicht anders zu beurteilen sind, als solche, bei denen sich nur geringfügigere, oft nur besonders liebevollem Studium sich offenbarende Abweichungen von der Norm finden, das glaube ich oben gezeigt zu haben. Die zukünftige Forschung wird vermutlich noch vielfach in dies Gebiet fallende Befunde bei Epileptikern zu erheben haben. Es wird sich dann wahrscheinlich herausstellen, daß es auf die spezielle, dem untersuchenden Pathologen sich darbietende, Ä u ßerung der gestörten Entwicklung nicht an kom mt. Denn nicht diese an sich ist es, die die erhöhte ,epileptische Reaktionsfähigkeit" des betroffenen Gehirns bedingt, sondern höchst wahrscheinlich mit ihr verknüpfte, weit feinere Abweichungen von der normalen Struktur der nervösen Elemente, die für uns vorläufig pathologisch-anatomisch noch nicht faßbar sind.

\section{Literaturverzeichnis.}

1. Alzhei mer, Ein Beitrag zur pathologischen Anatomie der Epilepsie. Monatsschrift f. Psych. u. Neurol. 4, 345. 1898.

2. Anton, Zur Kenntnis der Störungen im Oberflächenwachstum des menschlichen Großhirns. Zeitschr. f. Heilk., I. Mitt., y, 453, 1886; II. Mitt. 9, 237. 1888.

3. - Entwicklungsstörungen des Gehirns. Handb. d. pathol. Anat. d. Nervensystems 1904 , S. 417 , spez. S. $427 \mathrm{ff}$.

4. Astwazaturow, Über Epilepsie bei Tumoren des Schläfenlappens. Monatsschrift f. Psych. u. Neurol. 29, 342. 
5. Binswanger, Die klinische Stellung der sog. genuinen Epilepsie. Monats. schrift f. Neurol. u. Psych. 32, 369.

6. Bevan-Levis, zitiert nach Jakob (12).

6a. Bielschowsky, Über Mikrogyrie. Journ. f. Psychol. u. Neurol. 22, 1. 1915.

6b. - Epilepsie und Gliomatose. Journ. f. Psychol. u. Neurol. 21, 353. 1915.

7. Chiari, Über einen Fall von Mikrogyrie (Heschl) bei einem 13 monatigen idiotischen Knaben. Jahrb. f. Kinderheilk. Neue Folge 14, 219.1879, spez. S. 225.

8. Groz, Mikrogyrie und Balkenmangel im menschlichen Gehirn. Archiv f. Psych. 45, 605.

9. Heilbronner, Über die Auffassung und Bedeutung aphasischer Störungen bei Epileptikern. Centralbl, f. Nervenheilk. u. Psych. 28, 249. 1905.

10. Heschl, UUber die vordere graue Schläfenwindung des menschlichen Großhirns. Festschr. zur 25. Jubiläumsfeier d. Landesirrenanst. in Wien. Zitiert nach Ranke (28).

11. His, Die Entwicklung des menschlichen Gehirns während der ersten Monate. S. Hirzel. Leipzig 1904.

12. Jakob, Zur Pathologie der Epilepsie. Zeitschr. f. d. ges. Neur. u. Psych. 23, 1. 1914.

13. Jelgersma, Das Gehirn ohne Balken. Ein Beitrag zur Windungstheorie. Neurol. Centralbl. 1890, S. 162.

14. Kalischer, Über Mikrogyrie und Mikrophthalmie. Neurol. Centralbl. 1899, S. 398.

15. Kotschetkowa, Beitrag zur pathologischen Anatomie der Mikrogyrie und der Mikrocephalie. Archiv f. Psych. 34, 39. 1901.

15 a. Landsbergen, Über Balkenmangel. Zeitschr. f. d. ges. Neur. u. Psych. 11, 515. 1912.

16. Liebscher, Zur Kenntnis der sog. Mikrogyrie. Zeitschr. f. Heilk. 20, 357. 1899.

17. - Zur Kenntnis der Mikrogyrie nebst einigen Bemerkungen über die sog. Heterotopien im Rückenmark des Menschen. Zeitschr. f. Heilk. 2r, Neue Folge $\gamma, 219.1906$.

18. Marchand, Beschreibung dreier Mikrocephalengehirne nebst Vorstudien zur Anatomie der Mikrocephalie. Nova acta Acad. Caesar. Leop.-Carol. Germ. naturae curiosorum 53, 329. Halle 1889.

19. Matell, Ein Fall von Heterotopie der grauen Substanz in den beiden Hemisphären des Großhirns. Archiv f. Psych.25, 124. 1893.

20. Meine, Ein Beitrag zur Lehre von der echten Heterotopie grauer Hirnsubstanz. Archiv f. Psych. 30, 608.

21. Meschede, Über Neubildung grauer Hirnsubstanz in den Wandungen der Seitenventrikel usw. Allgem. Zeitschr. f. Psych. 21, 481. 1864.

22. v. Monakow, Über die Mißbildungen des Zentralnervensystems. Lubarsch Ostertags Ergebnisse 6, 513, spez. 555ff. 1899. Wiesbaden 1901.

23. - Über einen Fall von Mikrocephalie mit Sektionsbefund. Ber. südwestd. Vers. Archiv f. Psych. 31, 845. 1898.

24. Oppenheim, H., Über Mikrogyrie und eine infantile Form der Glossopharyngolabialparalyse. Neurol. Centralbl. 1895.

25. Oeconomakis, Über umschriebene mikrogyrische Verbildungen an der Großhirnoberfläche und ihre Beziehungen zur Porencephalie. Archiv f. Psych. 39, 676. 1905.

26. Probst, Über den Bau des vollständig balkenlosen Großhirns sowie über Mikrogyrie und Heterotopie der grauen Substanz. Archiv f. Psych. 34, 709. 1901 . 
27. Probst, Die Lehre von der Mikrocephalie und Makrogyrie. Archiv f. Psych. 38, 47. 1904.

28. Ranke, Beiträge zur Kenntnis der normalen und pathologischen Hirnrindenbildung. Zieglers Beiträge z. allg. Path. u. pathol. Anat. 47, 5l. 1910.

29. Redlich, Ưber Halbseitenerseheinungen bei der genuinen Epilepsie. Archiv f. Psych. 41, 567.

30. - Die klinische Stellung der sog. genuinen Epilepsie. Ref. erstattet auf der Tagung d. Ges. d. Nervenärzte in Hamburg 1912. Berlin 1913.

31. Reichert, Der Bau des menschlichen Gehirns, S. 76f., zitiert nach $R$ a n ke (28).

32. Roncoroni, Die Histologie der Stirnlappenrinde bei Verbrechern und Epileptikern. Wiener klin. Rundschau 1897, Nr. 6, 7, 8. Zitiert nach Jakob (12).

33. Rondoni, Beitrag zum Studium der Entwicklungskrankheiten des Gehirns. Archiv f. Psych. 45, 1004 (spez. Teil II). 1909.

34. Steiner, Epilepsie und Gliose. Archiv f. Psych. 46, 1091.

35. Stern, Die psychischen Störungen bei Hirntumoren und ihre Beziehungen zu den durch Tumorwirkung bedingten diffusen Hirnveründerungen. Archiv f. Psych. 54, 565. 1914.

36. Stransky, zitiert nach Redlich (30).

37. Turner, Epilepsy, a study of the idiopathic disease. London 1907. Zitiert nach Jakob (12).

38. Virchow, Zur pathologischen Anatomie des Gehirns. II. Teil: Heterotopie der grauen Hirnsubstanz. Virchows Archiv 38, 138. 1867.

39. Vogt, Heinr., Über die Anatomie, das Wesen, die Entstehung mikrocephaler Mißbildungen. Arbeiten a. d. hirnanat. Inst. d. Univ. Zürich 1905, H. 1.

40. - Über Ziele und Wege der teratologischen Hirnforschungsmethode. Monats. schrift f. Psych. u. Neurol. 1\%, 337 u. 415. 1905.

41. Zingerle, Ein Fall von umschriebener Störung im Oberflächenwachstum des Gehirns. Archiv f. Psych. 36, 97.

\section{Erklärung der Tafeln II u. III.}

Fig. 1: Toluidinblau. Fall 1. Carla M. Anordnung der Ganglienzellen: in der oberflächlichen Rinde (a) senkrecht zur Oberfläche, in der tiefen Rinde (b) parallel zur Oberfläche. (Die Photographie gibt nur einen Ausschnitt, weil bei einem Maßstab, der beide Rinden in toto erkennen ließe, die Richtung der Ganglienzellen nicht genügend deutlich sein würde).

Fig. 2-4: Weigertsche Gliafaserfärbung. Oberflächliche Gliose.

Fig. 2: Fall I. Carla M.

Fig. 3: Fall II. Lina J.: Stirnhirn.

Fig. 4: Fall II. Lina J.: Linker Schläfenlappen. 